\title{
Pharmacogenetics of Vascular Risk Factors in Alzheimer's Disease
}

\author{
Ramón Cacabelos ${ }^{1, *}$, Arun Meyyazhagan 1,2, Juan C. Carril 1,2, Pablo Cacabelos 1,2 \\ and Óscar Teijido 1,2 \\ 1 EuroEspes Biomedical Research Center, Institute of Medical Science and Genomic Medicine, Bergondo, \\ 15165 La Coruña, Spain; cytogenetics@euroespes.com (A.M.); genomica@euroespes.com (J.C.C.); \\ asistentedireccion@euroespes.com (P.C.); epigenetica@euroespes.com (Ó.T.) \\ 2 Chair of Genomic Medicine, Continental University Medical School, Huancayo 12000, Peru \\ * Correspondence: rcacabelos@euroespes.com; Tel.: +34-981-780505; Fax: +34-981-780511
}

Received: 23 October 2017; Accepted: 27 December 2017; Published: 3 January 2018

\begin{abstract}
Alzheimer's disease (AD) is a polygenic/complex disorder in which genomic, epigenomic, cerebrovascular, metabolic, and environmental factors converge to define a progressive neurodegenerative phenotype. Pharmacogenetics is a major determinant of therapeutic outcome in AD. Different categories of genes are potentially involved in the pharmacogenetic network responsible for drug efficacy and safety, including pathogenic, mechanistic, metabolic, transporter, and pleiotropic genes. However, most drugs exert pleiotropic effects that are promiscuously regulated for different gene products. Only $20 \%$ of the Caucasian population are extensive metabolizers for tetragenic haplotypes integrating $C Y P 2 D 6-C Y P 2 C 19-C Y P 2 C 9-C Y P 3 A 4 / 5$ variants. Patients harboring CYP-related poor (PM) and/or ultra-rapid (UM) geno-phenotypes display more irregular profiles in drug metabolism than extensive (EM) or intermediate (IM) metabolizers. Among 111 pentagenic ( $A P O E-A P O B-A P O C 3-C E T P-L P L)$ haplotypes associated with lipid metabolism, carriers of the $\mathrm{H} 26$ haplotype (23-TT-CG-AG-CC) exhibit the lowest cholesterol levels, and patients with the H104 haplotype (44-CC-CC-AA-CC) are severely hypercholesterolemic. Furthermore, APOE, NOS3, $A C E, A G T$, and CYP variants influence the therapeutic response to hypotensive drugs in $\mathrm{AD}$ patients with hypertension. Consequently, the implementation of pharmacogenetic procedures may optimize therapeutics in AD patients under polypharmacy regimes for the treatment of concomitant vascular disorders.
\end{abstract}

Keywords: Alzheimer's disease; anti-dementia drugs; APOE; atorvastatin; cholesterol; CYP haplotypes; Enalapril; hypertension; LipoEsar; pharmacogenetics

\section{Introduction}

Alzheimer's disease (AD) is a complex disorder in which genomic, epigenomic, cerebrovascular, metabolic, and environmental factors are potentially involved [1-5]. About 85\% of patients over 75 years of age exhibit an important cerebrovascular component in their neuropathological phenotype $[4,5]$. Furthermore, AD patients present concomitant disorders including hypertension $(20-30 \%)$, overweightness or obesity $(20-40 \%)$, diabetes $(20-25 \%)$, hypercholesterolemia $(>40 \%)$, anemia $(>20 \%)$, metabolic deficiencies $(>15 \%)$, atherosclerosis $(>60 \%)$, cardiovascular disease $(>40 \%)$, and cerebrovascular damage (60\%), which require additional treatments [6-8]. Most of these clinical conditions and biochemical anomalies show gender differences and may contribute to accelerating the dementia process. The pharmacological treatment of these concomitant pathologies adds complexity and risks to the multifactorial therapeutic intervention employed in patients with dementia. Of major relevance is the treatment of diabetes, hypertension, dyslipidemia, and cardiovascular, cerebrovascular, and neuropsychiatric disorders. AD patients receive a higher burden of psychotropic 
drugs, especially neuroleptics, antidepressants, and benzodiazepines, than their age-matched counterparts [9]. The chronic treatment of diverse illnesses with pleiotropic drugs increases the risk of drug interactions and toxicity, aggravating the clinical condition of the demented patient. In this context, the incorporation of pharmacogenetic protocols into clinical practice is fundamental to minimize drug-drug interactions and adverse drug reactions (ADRs) and to optimize the global therapeutic outcome, avoiding deleterious effects on mental function and cognition $[2,3,6-8,10,11]$.

Major determinants of therapeutic outcome in AD include age- and sex-related factors, pathogenic phenotype, concomitant disorders, treatment modality and polypharmacy, and pharmacogenetics. Different categories of genes are potentially involved in the pharmacogenetic network responsible for drug efficacy and safety. Pathogenic, mechanistic, metabolic, transporter, and pleiotropic genes represent the major genetic determinants of response to treatment in $\mathrm{AD}[2,6,8,10]$. By-products of these genes are integrated in transcriptomic, proteomic, and metabolic networks, which are disrupted in $\mathrm{AD}$ and represent potential targets for therapeutic intervention $[8,10,11]$.

$\mathrm{AD}$ patients may take over ten different drugs/day for the treatment of dementia-related symptoms (memory deterioration, behavioral changes, and functional decline) and concomitant disorders (cardiovascular, cerebrovascular, parkinsonian, epileptic, hypertensive, dyslipidemic, and metabolic problems). The older the patient, the higher the number of drugs taken in polypharmacy regimes [9]. Approximately $30-40 \%$ of current drugs may contribute to abnormal amyloidogenesis [12]. The co-administration of several drugs may cause ADRs, which in some cases require hospitalization. The prevalence of potentially inappropriate medication (PIM) is around 50\% in some European cohorts. Cerebral vasodilators are the most widely used class of PIM, accounting for $24.0 \%$ of all prescriptions, followed by atropinic drugs and long half-life benzodiazepines. Atropinic drugs are associated with cholinesterase inhibitors in $16 \%$ of patients. In over $20 \%$ of the patients, behavioral deterioration and psychomotor function can be severely altered by polypharmacy [13]. The principal causes of these iatrogenic effects are the inappropriate combination of drugs, and the genomic background of the patient, responsible for his/her pharmacogenomic outcome.

The cerebrovascular component of $\mathrm{AD}$ is a contributing factor to aggravate disease progression [4,5]; cerebrovascular atherosclerosis may coexist with $\mathrm{AD}$ [14]; and, in late-onset AD, vascular risk factors are associated with earlier clinical manifestation [15]. Furthermore, the co-administration of drugs for the treatment of vascular risk factors present in AD patients increases the ADR rate and exerts deleterious effects on brain function and cognition. Typical examples of this are heart disorders, hypercholesterolemia, and hypertension. Some drugs for the treatment of concomitant cardio/cerebrovascular disorders may interact with conventional anti-dementia drugs. At the present time, the only way to minimize the impact of potential ADRs in AD patients under polypharmacy protocols is the implementation of pharmacogenetic protocols [16,17].

\section{Genetic Determinants of the Pharmacogenomic Network}

Pharmacogenomics accounts for $60-90 \%$ variability in the pharmacokinetics and pharmacodynamics of common drugs in the market. The modest effect (and ADRs) of current AD drugs are in part due to their pharmacogenomic profile, since over $80 \%$ of $\mathrm{AD}$ patients are deficient metabolizers $[3,8,10,17]$. The genes involved in the pharmacogenomic response to drugs in dementia fall into five major categories: pathogenic genes associated with AD-related neurodegeneration (APP, PSEN1, PSEN2, $M A P T, A P O E$, and $>600$ susceptibility genes), mechanistic genes associated with the mechanisms of action of drugs (receptors, enzymes), metabolic genes encoding enzymes involved in Phase I and Phase II metabolizing reactions in liver and other tissues [Cytochrome P450 (CYPs), UDP Glucuronosyl Transferases (UGTs), and N-Acetyl Transferases (NATs)], transporter genes encoding transporter proteins [ATP-Binding Cassette Transporters (ABCs) and Solute Carrier Transporters (SLCs)], and pleiotropic genes with multi-locative effects in different pathogenic cascades [3,6,8,10,16-20].

Although the APP, PSEN1, PSEN2, and MAPT genes are considered major pathogenic genes for $\mathrm{AD}$ and classic tauopathies [18-20], mutations in these genes represent less than $5 \%$ of the AD 
population; consequently, their influence on $\mathrm{AD}$ pharmacogenetics associated with conventional anti-dementia drugs is quantitatively negligible; not so in the case of immunotherapy or secretase inhibitors/modulators addressing amyloid- $\beta(A \beta)$ deposition. In this case, gene mutations affect the amyloidogenic and/or tauopathic phenotypes and, consequently, the outcome of pharmacological interventions may be affected by particular genotypes. Most anti-AD vaccines (active and passive immunization) are based on transgenic models with APP, PSEN1, and PSEN2 mutants [21,22]. Depending on the transgenic model, the phenotypic expression of $A \beta$ deposition may vary and the therapeutic effects of immunization may be different [23].

To date, the most influential gene in AD pharmacogenetics is the APOE gene $[2,6-8,10,16,17,24]$. The vast majority of pharmacogenetic studies in $\mathrm{AD}$ have been performed with susceptibility genes $(A P O E)$ and metabolic genes (CYPs) $[8,10,24,25]$. In general terms, APOE-3 carriers tend to be the best responders to conventional antidementia drugs (donepezil, rivastigmine, galantamine, and memantine), and $A P O E-4$ carriers are the worst responders to different treatments $[6-8,10,14,17,24,25]$. The association of the TOMM40-L/L genotype with the APOE-4/4 genotype yields a haplotype $(4 / 4-\mathrm{L} / \mathrm{L})$ that is responsible for early onset of the disease, a faster cognitive decline, and a poor response to treatment $[7,8,16,17]$. CYP2D6 variants also influence the therapeutic outcome, with extensive metabolizers as the best responders, followed by intermediate metabolizers; whereas poor and ultra-rapid metabolizers exhibit a deficient response to drugs in terms of efficacy and safety $[6,10,16,17,24-26]$. Those CYP2D6 extensive metabolizers (EMs) who harbor an APOE-4/4 genotype are poor responders to conventional treatments, reflecting the negative influence that the APOE-4 allele exerts on the pharmacogenetic outcome in AD patients $[6,10,16,17,24-26]$.

Other recent pharmacogenetic studies with pathogenic or mechanistic genes indicate that the response to cholinesterase inhibitors (AChEIs) can be modulated by genes associated with the cholinergic system. Genetic variants in CHRNA7, encoding a subunit of the acetylcholine receptor $(\alpha 7-n A C h R)$, are associated with the response to AChEIs. Pharmacogenetic studies in an Italian cohort with two single nucleotide polymorphisms (SNPs) (rs6494223 and rs8024987) in the CHRNA7 gene revealed that the rs6494223 variant may affect response to AChEIs [27]. Variability in the clinical response to AChEIs is also associated with 2 SNPs in the intronic region of CHAT rs2177370 and rs3793790 [28]. The CHRNA7 T allele (rs6494223) also associates with a better response to AChEIs, and there is further confirmation that $A P O E-4$ carriers are the worst responders to conventional AChEIs [29]. Butyrylcholinesterase (BChE) activity increases with disease progression and may replace acetylcholinesterase function. The $\mathrm{BChE} \mathrm{K-variant} \mathrm{is} \mathrm{associated} \mathrm{with} \mathrm{lower} \mathrm{acetylcholine-hydrolyzing}$ activity and with a poor response to donepezil, similar to that observed in APOE-4 carriers [30]. A genome-wide association study in 176 AD patients identified 2 SNPs with apparent response to treatment; one SNP (rs6720975A) maps in the intronic region of PRKCE, and the another one (rs17798800A) is an intergenic segment acting as a cis-regulator of NBEA [31]. Old studies identified SNPs in Phase II reactions enzymes, such as gluthatione S-transferase theta, to be associated with the hepatotoxic effects of tacrine [32]. Many other variants influence the therapeutic response to AChEIs and combination treatments in $\mathrm{AD}[2,3,7,8,10,16,17,24-26]$.

All these genes involved in the pharmacogenomic network are under the influence of the epigenetic machinery (DNA methylation, histone/chromatin modifications, microRNA (miRNA) regulation) conditioning their expression and the efficiency of their drug-metabolizing products (enzymes, transporters) $[26,33,34]$.

\section{Drug Metabolism}

Over $70 \%$ of $A D$ patients are deficient metabolizers for the $C Y P 2 D 6 / 2 C 19 / 2 C 9$ trigenic cluster; and for the CYP2D6/2C19/2C9/3A4 tetragenic cluster, more than $80 \%$ of the patients exhibit a deficient metabolizer geno-phenotype [3,17]. These four CYP genes encode enzymes responsible for the metabolism of $60-80 \%$ of drugs of current use, showing ontogenic-, age-, sex-, circadian- and ethnic-related differences $[10,24,35,36]$. CYP2D6 enzymes metabolize over 900 different drugs 
(371 substrates, 300 inhibitors, and 18 inducers). CYP2C9 enzymes metabolize over 600 drugs (311 substrates, 375 inhibitors, and 41 inducers). Nearly 500 drugs are metabolized via CYP2C19 enzymes (281 substrates, 263 inhibitors, and 23 inducers). CYP3A4 and 3A5 enzymes metabolize over 1900 drugs (1033 substrates, 696 inhibitors, and 241 inducers) [36].

The distribution and frequency of CYP2D6 genotypes are very similar in the general population (GP) and in $\mathrm{AD}$, with the exception of the CYP2D6-*3/*4 genotype, which is absent in AD samples [17]. In the GP, CYP2D6 extensive metabolizers (EMs) account for $58.85 \%$, whereas intermediate metabolizers (IMs) account for 31.11\%, poor metabolizers (PMs) $4.49 \%$, and ultra-rapid metabolizers (UMs) 5.55\% [8,10,17]. In AD, EMs, IMs, PMs, and UMs represent $57.54 \%, 31.01 \%, 5.49 \%$, and 5.96\%, respectively [17]. There is an accumulation of AD-related genes of risk in PMs and UMs. EMs and IMs are the best responders, and PMs and UMs are the worst responders to a combination therapy with AChEIs, neuroprotectants, and vasoactive substances [2,10,37]. The pharmacogenetic response in AD appears to be dependent upon the networking activity of genes involved in drug metabolism and genes associated with AD pathogenesis [2,6,10,16,17,38]. By phenotypes, in the GP, CYP2C9-PMs represent 4.82\%, IMs 33.83\%, and EMs 61.35\%. In AD, PMs, IMs, and EMs represent 4.76\%, 34.87\%, and 60.37\%, respectively $[8,10,17]$. The frequencies of the CYP2C19 geno-phenotypes in the GP are CYP2C19-EMs (74.11\%), CYP2C19-IMs (24.43\%), and CYP2C19-PMs (1.46\%) [17]. EMs, IMs, and PMs account for $75.41 \%, 23.56 \%$, and $1.03 \%$, respectively, in $\mathrm{AD}[8,10,16,17]$. Concerning CYP3A4/5 polymorphisms in $\mathrm{AD}, 83.84 \%$ of the cases are EMs $\left(C Y P 3 A 5^{*} 3{ }^{*} 3\right), 14.62 \%$ are IMs $\left(C Y P 3 A 5^{*} 1{ }^{*} 3\right)$, and $1.54 \%$ are RMs $\left(C Y P 3 A 5^{*} 1 /{ }^{*}\right)$, whereas, in the GP, EMs, IMs, and RMs represent $82.17 \%, 16.48 \%$, and $1.35 \%$, respectively [17].

Tetragenic haplotypes integrating CYP2D6, CYP2C9, CYP2C19, and CYP3A4/5 variants yield 156 genotypes (Figure 1). The most frequent haplotype is H3 (1/1-1/1-1/1-3/3) (20.87\%), representing full extensive metabolizers, and only 17 haplotypes exhibit a frequency higher than $1 \%$ in the Iberian population (Figure 2). In addition to $\mathrm{H} 3$, the most frequent haplotypes $(>2 \%)$ are $\mathrm{H} 55(1 / 4-1 / 1-1 / 1-1 / 3)$ (8.41\%), H26 (1/1-1/2-1/1-3/3) (8.07\%), H4 (1/1-1/1-1/2-3/3) (8.07\%), H58 (1/4-1/1-1/2-3/3) (3.99\%), H72 (1/4-1/2-1/1-3/3) (3.82\%), H2 (1/1-1/1-1/1-1/3) (3.74\%), H9 (1/1-1/1-1/3-3/3) (3.57\%), and H38 $(1 \mathrm{xN} / 1-1 / 1-1 / 1-3 / 3)(2.46 \%)$ (Figure 2). Among Caucasians, about $80 \%$ of the population is deficient for the biotransformation of current drugs metabolized via CYP2D6-2C9-2C19-3A4 enzymes [17].

\section{Tetragenic (CYP2D6-CYP2C19-CYP2C9-CYP3A4/5) Haplotypes}

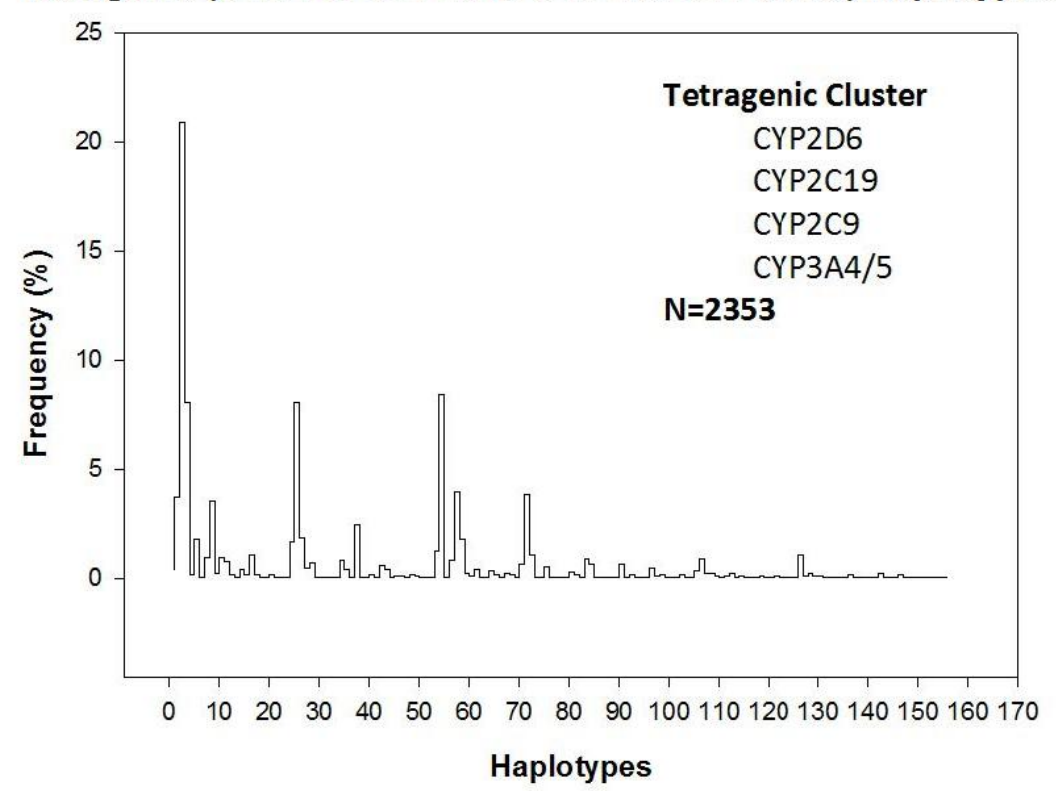

Figure 1. Tetragenic (CYP2D6-CYP2C19-CYP2C9-CYP3A4/5) haplotypes in the Spanish population. 


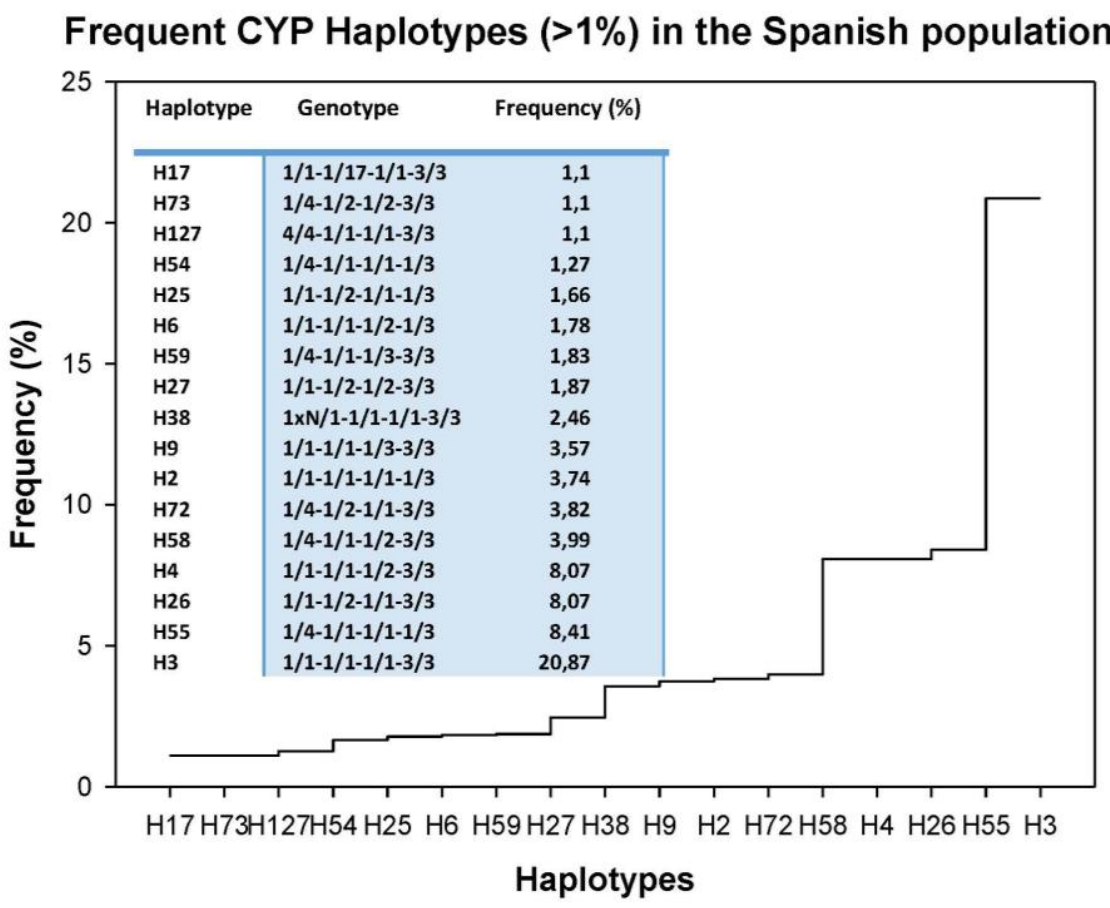

Figure 2. Frequent tetragenic CYP2D6-CYP2C19-CYP2C9-CYP3A4/5 haplotypes $(>1 \%)$ in the Spanish population.

\section{Genetic Determinants of Lipid Metabolism and Vascular Function}

Among hundreds of genes potentially involved in AD pathogenesis and concomitant disorders (cardiovascular and cerebrovascular disorders, hypercholesterolemia, and hypertension), at least four categories of genes deserve special attention: (i) genes associated with lipid metabolism $(A P O B$, $A P O C 3, A P O E, C E T P$, and $L P L$ ), (ii) genes associated with endothelial function and hypertension (NOS3, ACE, and AGT); (iii) genes associated with immune function and inflammation (IL1B, IL6, IL6R, and TNFA); and (iv) genes associated with thrombosis and coagulation ( $F 2, F 5$, and MTHFR) $[17,36,39]$.

Although differences in genotype distribution and frequencies of all these genes between patients with $\mathrm{AD}$ and control subjects are negligible, except in the case of APOE [39] (Figures 3 and 4), some of them may influence the pharmacogenetic outcome in the treatment of major risk factors for dementia, such as hypercholesterolemia, cardiovascular disorders, and hypertension [39-43]. Furthermore, many of these genes interact in pathogenic cascades contributing to alter blood pressure, brain cholesterol, and $\mathrm{A} \beta$ metabolism, subsequently accelerating neuronal death in $\mathrm{AD}$. A clear example is the angiotensin-converting enzyme (ACE), which degrades $\mathrm{A} \beta$, and $\mathrm{ACE}$ inhibitors that contribute to slow down cognitive decline. In fact, some SNPs in the ACE gene (rs1800764 and rs4291) are associated with cognitive modification and therapeutic response to anti-hypertensive treatment with ACE inhibitors [44]. 


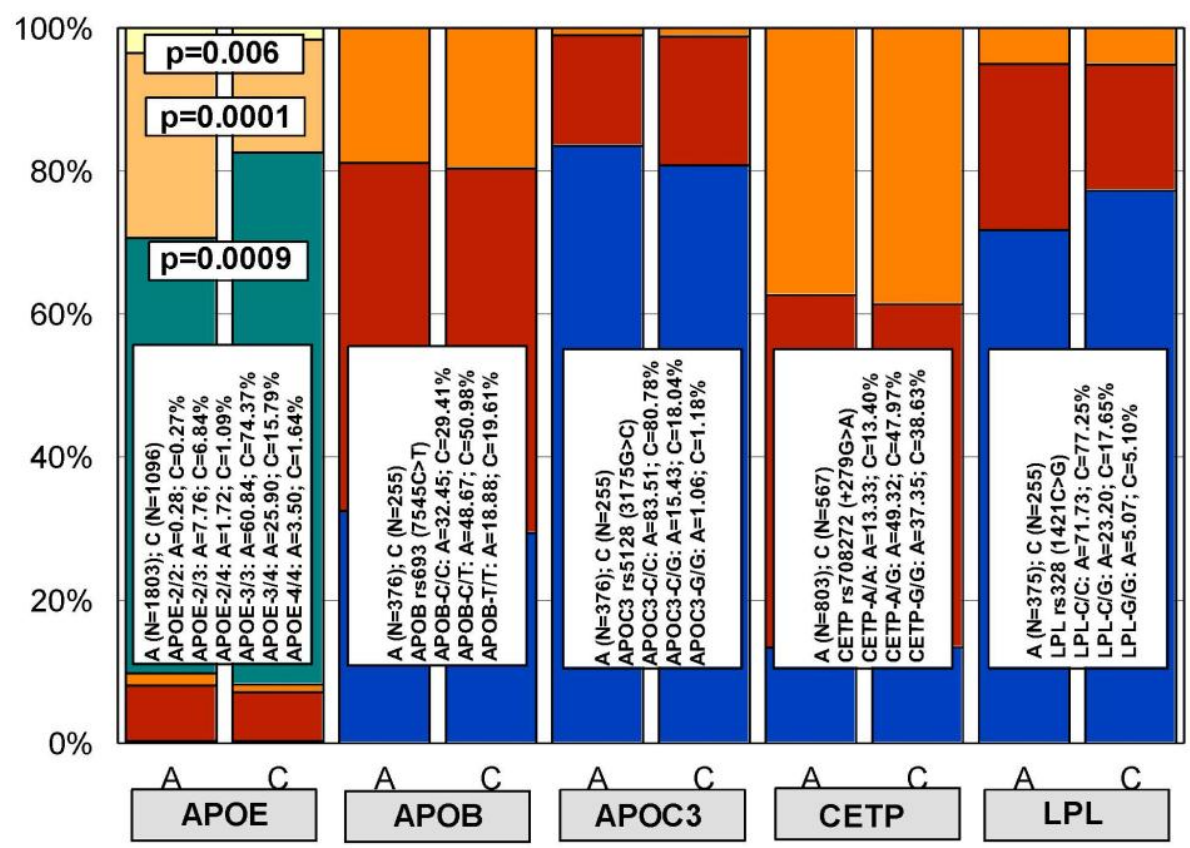

Figure 3. Distribution and frequency of polymorphic variants of the $A P O E, A P O B, A P O C 3, C E T P$, and $L P L$ genes in the general population and in patients with Alzheimer's disease. A: Alzheimer's disease; C: Control Population. Color codes: Blue: APOE-2/2, APOB-C/C, APOC3-C/C, CETP-A/A, LPL-C/C; Red: APOE-2/3, APOB-C/T, APOC3-C/G, CETP-A/G, LPL-C/G; Orange: APOE-2/4, APOB-T/T, APOC3-G/G, CETP-G/G, LPL-G/G; Green: APOE-3/3; Golden: APOE-3/4; Yellow: APOE-4/4.

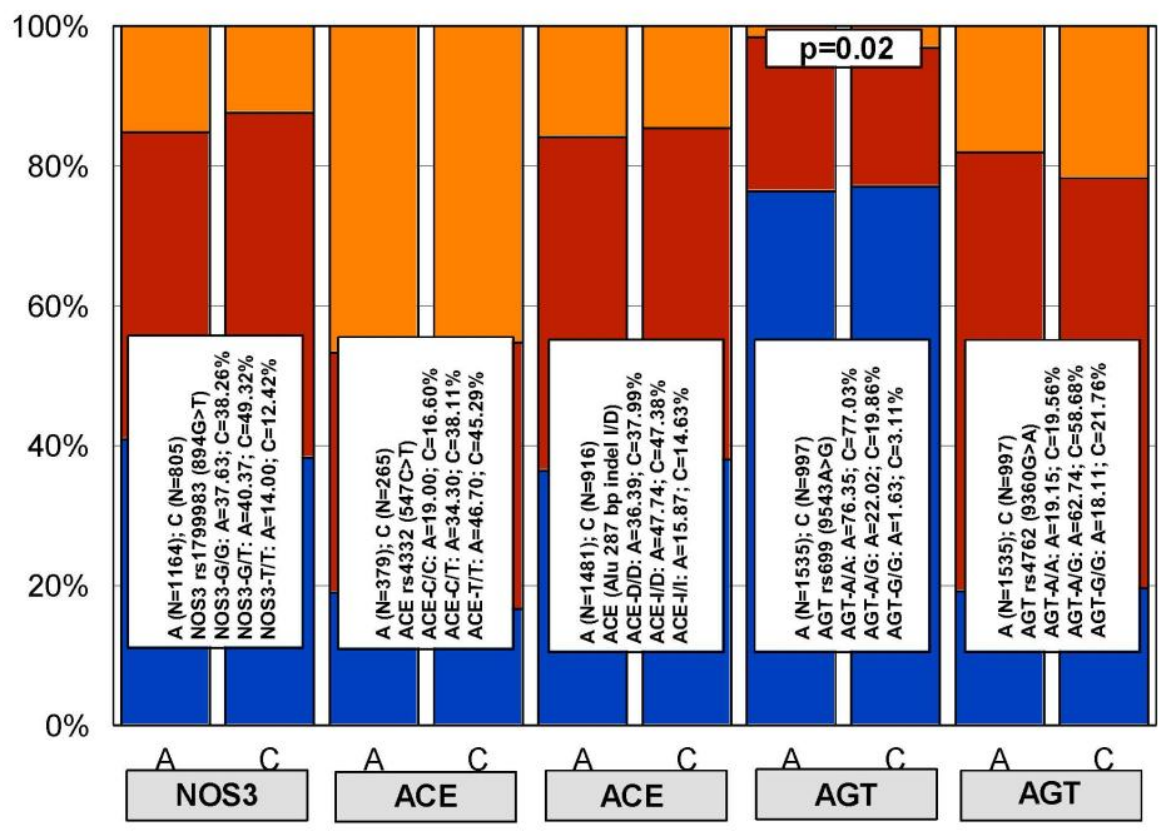

Figure 4. Distribution and frequency of polymorphic variants of the NOS3, $A C E$, and $A G T$ genes in the general population and in patients with Alzheimer's disease. Color codes: Blue: NOS3-G/G, $A C E-C / C$, ACE-D/D, AGT-A/A; Red: NOS3-G/T, ACE-C/T, ACE-I/D, AGT-A/G; Orange: NOS3-T/T, ACE-C/T, ACE-I/I, AGT-G/G. 


\section{Pharmacogenetics of Hypercholesterolemia in Alzheimer's Disease}

Alterations in cholesterol $(\mathrm{CHO})$ metabolism are involved in AD pathogenesis and over $40 \%$ of $\mathrm{AD}$ patients are hypercholesterolemic. High $\mathrm{CHO}$ levels are also associated with vascular dementia [45]. It has been postulated that statins, prescribed as lipid-lowering drugs to patients at risk for cardiovascular conditions, may be beneficial in AD [46-48]. Statins are currently used in AD [9]; however, clinical evidence shows conflicting results and poor benefits [49-52]. The potential beneficial effect of statins and reduction in AD risk varied across statin molecules, sex, and race/ethnicity [53]. Other studies indicate that simvastatin exacerbates amyloid angiopathy [54]. In contrast, lovastatin might reduce $A \beta$ levels in humans [55]. Some mechanisms by which simvastatin and atorvastatin might facilitate amyloid- $\beta$-protein degradation would be by increasing neprilysin secretion from astrocytes through activation of Mitogen-activated protein kinase/extracellular signal-regulated kinases (MAPK/Erk1/2) pathways [56], regulation of cholesterol in lipid rafts, suppression of inflammation, and inhibition of oxidative stress [47]. The pleiotropic effects of statins (simvastatin, atorvastatin, cerivastatin, fluvastatin, pravastatin, and rosuvastatin) (Table 1 are apparently APOE-independent; however, APOE is a fundamental factor in the regulation of lipid metabolism, and APOE variants influence the therapeutic effect of most hypolipemic compounds, including statins $[8,10,17,36]$.

Cognitive deterioration shows a clear age-dependent profile in $\mathrm{AD}$, with an average decline of 3-5 points/year (Mini-Mental State Examination (MMSE) score); however, total CHO levels do not appear to affect mental deterioration in AD [17]. Blood lipid levels also show a moderate age-dependent profile. In the GP, CHO levels tend to increase with age, reaching a plateau at 60-70 years of age and declining thereafter; however, $\mathrm{CHO}$ levels in $\mathrm{AD}$ tend to diminish in an age-related fashion, but in age-matched samples $\mathrm{CHO}$ levels tend to be higher in AD as compared to GP [17].

In previous studies, we have demonstrated that $A P O E$ and $C Y P$ variants $(2 \mathrm{D} 6,2 \mathrm{C} 9,2 \mathrm{C} 19$, 3A4/5) influence basal $\mathrm{CHO}$ levels and the therapeutic response of AD patients to hypolipemic compounds $[10,14,17]$. In a larger study with 1345 hypercholesterolemic $A D$ patients $(\mathrm{CHO}>220 \mathrm{mg} / \mathrm{dL})$, we investigated the pharmacogenetics of cholesterol response to Atorvastatin (10-20 mg/day) plus SardiLipin (E-SAR-94010; LipoEsar ${ }^{\circledR}$; Ebiotec, Bergondo, Spain) (500 mg/day), a nutraceutical compound of the ProteoLipin/LipoFishin family [57], with lipid-lowering effects and anti-atherosclerotic and neuroprotective properties (Patent ID: P9602566) $[10,36,58]$. In the whole sample, the response rate (RR) was $78.96 \%$ responders ( $\mathrm{CHO}<$ baseline levels) and $21.04 \%$ non-responders $(\mathrm{CHO} \geq$ baseline levels) after one month of treatment (Figure 4). APOE-related basal CHO levels are significantly different, with females showing higher $\mathrm{CHO}$ levels than males; however, females and males responded similarly to the hypolipemic treatment [17]. The stratification of patients according to their APOE, $A P O B, A P O C 3, C E T P$, and $L P L$ genotypes showed no genotype-related differences at basal $\mathrm{CHO}$ levels, except in the case of $A P O E-4$ carriers, where the highest baseline levels of $\mathrm{CHO}$ were found in APOE-4/4 carriers [17] (Figure 3).

In a selected group of $933 \mathrm{AD}$ patients, we constructed a pentagenic haplotype integrating all possible variants of the $A P O E+A P O B+E P O C 3+C E T P+L P L$ genes and identified 111 haplotypes (H) (Figure 5) with differential basal CHO levels (Figure 6). About $75 \%$ of these haplotypes in the AD population have a frequency below $1 \%, 10 \%$ have a frequency between $1 \%$ and $2 \%, 8 \%$ have a frequency between $2 \%$ and $5 \%$, and only $4 \%$ of the haplotypes are present in more than $5 \%$ of AD patients [17]. The haplotypes most frequently found are H55 (33-CT-CC-AG-CC) (8.79\%), H58 (33-CT-CC-GG-CC) and H37 (33-CC-CC-AG-CC) (7.07\%). Haplotypes H104 (44-CC-CC-AA-CC) $(0.11 \%), H 110$ (44-TT-CC-AG-CG) $(0.11 \%)$ and H98 (34-TT-CC-AA-CG) $(0.11 \%)$ showed the highest CHO levels, and the lowest levels corresponded to haplotypes H26 (23-TT-CG-AG-CC) (0.11\%), H8 (23-CC-CG-AG-CC) (0.21\%), H50 (33-CC-GG-AG-CC) (0.21\%), and H63 (33-CT-CG-AA-GG) (0.11\%) [17] (Figure 6). 
Table 1. Pharmacological properties and pharmacogenetics of statins.

\begin{tabular}{|c|c|c|}
\hline \multicolumn{3}{|c|}{ HMG CoA Reductase Inhibitors } \\
\hline Drug & Properties & Pharmacogenetics \\
\hline & $\begin{array}{l}\text { Name: ATORVASTATIN CALCIUM } \\
\text { Molecular Formula: } \mathrm{C}_{66} \mathrm{H}_{68} \mathrm{CaF}_{2} \mathrm{~N}_{4} \mathrm{O}_{10} \\
\text { Molecular Weight: } 1155.341726 \mathrm{~g} / \mathrm{mol} \\
\text { Mechanism: Inhibits HMG-CoA reductase, resulting in } \\
\text { a compensatory increase in the expression of LDL } \\
\text { receptors on hepatocyte membranes and a stimulation } \\
\text { of LDL catabolism. } \\
\text { Effect: Anticholesteremic Agent; HMG-CoA Reductase } \\
\text { Inhibition; Apolipoprotein B reduction; Triglyceride } \\
\text { reduction; Anti- atherosclerotic; Heart-health effects. }\end{array}$ & 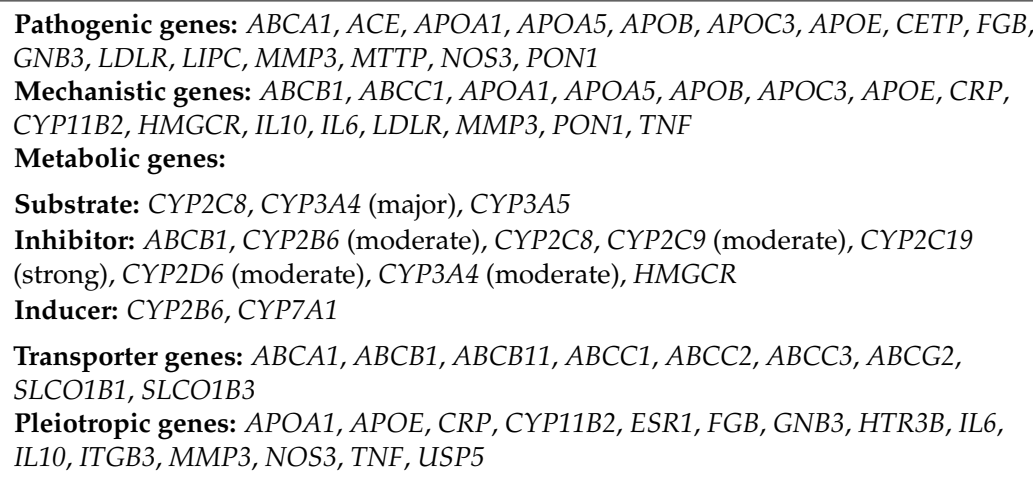 \\
\hline & $\begin{array}{l}\text { Name: FLUVASTATIN SODIUM } \\
\text { Molecular Formula: } \mathrm{C}_{24} \mathrm{H}_{25} \mathrm{FNNaO}_{4} \\
\text { Molecular Weight: } 433.447772 \mathrm{~g} / \mathrm{mol} \\
\text { Mechanism: Acts by competitively inhibiting HMGCR, } \\
\text { the enzyme that catalyzes reduction of HMG-CoA to } \\
\text { mevalonate. HDL is increased while total, LDL and } \\
\text { VLDL cholesterols, apolipoprotein B, and plasma } \\
\text { triglycerides are decreased. } \\
\text { Effect: Anticholesteremic Agent; HMG-CoA Reductase } \\
\text { Inhibition; Heart-health effects; Antineoplastic activity; } \\
\text { Immune response modulation }\end{array}$ & $\begin{array}{l}\text { Pathogenic genes: } A B C A 1, A C E, A P O A 1, A P O A 5, A P O B, A P O C 3, A P O E, C E T P \text {, } \\
C Y P 7 A 1, L D L R, L I P C, L P L, N O S 3, P P A R D, P O N 1 \\
\text { Mechanistic genes: } A P O A 1, A P O B, H M G C R, L P L, P O N 1 \\
\text { Metabolic genes: } \\
\text { Substrate: CYP1A1 (minor), CYP2C8 (minor), CYP2C9 (major), CYP2D6 (minor), } \\
\text { CYP3A4 (minor), UGT1A3 } \\
\text { Inhibitor: CYP1A2 (weak), CYP2C8 (weak), CYP2C9, (moderate), CYP2C19 } \\
\text { (moderate), CYP2D6 (moderate), CYP3A4 (moderate), HMGCR } \\
\text { Inducer: CYP2B6, CYP3A4, } \\
\text { Transporter genes: } A B C A 1, A B C B 1, A B C B 11, A B C C 2, A B C G 2, S L C 15 A 1, S L C 22 A 8 \text {, } \\
\text { SLCO1B1, SLCO1B3, SLCO2B1 } \\
\text { Pleiotropic genes: } A C E, A P O A 1, A P O E, N O S 3, N R 112, N R 113, P P A R D, U S P 5\end{array}$ \\
\hline & $\begin{array}{l}\text { Name: LOVASTATIN } \\
\text { Molecular Formula: } \mathrm{C}_{24} \mathrm{H}_{36} \mathrm{O}_{5} \\
\text { Molecular Weight: } 404.53964 \mathrm{~g} / \mathrm{mol} \\
\text { Mechanism: Acts by competitively inhibiting } \\
\text { HMG-CoA reductase, enzyme which catalyzes } \\
\text { rate-limiting step in cholesterol biosynthesis. } \\
\text { Effect: Anticholesteremic Agent; HMG-CoA Reductase } \\
\text { Inhibition; Heart-health effects; Antineoplastic activity. }\end{array}$ & $\begin{array}{l}\text { Pathogenic genes: } A B C A 1, A P O A 1, A P O A 5, A P O B, A P O C 3, C E T P, L D L R, L I P C, L P L \\
\text { Mechanistic genes: } A P O A 1, A P O B, C E T P, H M G C R, L D L R \\
\text { Metabolic genes: } \\
\text { Substrate: } C Y P 3 A 4 \text { (major), CYP3A5, UGT1A3. } \\
\text { Inhibitor: } A B C B 1, C Y P 2 C 8, C Y P 2 C 9 \text { (weak), CYP2C19 (strong), CYP2D6 (weak), } \\
\text { CYP3A4 (weak), HMGCR, KCNH2 } \\
\text { LInducer: CYP2B6, CYP7A1 } \\
\text { Transporter genes: } A B C A 1, A B C B 1, A B C B 11, A B C C 2, A B C G 2, K C N H 2 \text {, } \\
\text { SLCO1B1, SLCO1B3 } \\
\text { Pleiotropic genes: TP53, USP5 }\end{array}$ \\
\hline
\end{tabular}


Table 1. Cont

\begin{tabular}{|c|c|c|}
\hline \multicolumn{3}{|c|}{ HMG CoA Reductase Inhibitors } \\
\hline Drug & Properties & Pharmacogenetics \\
\hline & $\begin{array}{l}\text { Name: PITAVASTATIN } \\
\text { Molecular Formula: } \mathrm{C}_{25} \mathrm{H}_{24} \mathrm{FNO}_{4} \\
\text { Molecular Weight: } 421.460763 \mathrm{~g} / \mathrm{mol} \\
\text { Mechanism: Works to control the synthesis of } \\
\text { cholesterol via competitive inhibition of the liver } \\
\text { enzyme, HMG-CoA reductase. As a result, } \\
\text { a ompensatory increase in LDL-receptor expression } \\
\text { can be observed which facilitates an increase } \\
\text { LDL catabolism. } \\
\text { Effect: Anticholesteremic Agent; HMG-CoA Reductase } \\
\text { Inhibition; Heart-health effects. }\end{array}$ & $\begin{array}{l}\text { Pathogenic genes: } A P O B, L D L R \\
\text { Mechanistic genes: } A P O B, H M G C, L D L R, P P A R G, P O N 1, V C A M 1 \\
\text { Metabolic genes: } \\
\text { Substrate: } A B C B 1, C Y P 2 C 8 \text { (minor), CYP2C9 (minor), CYP3A4 (minor), SLCO1B1, } \\
\text { UGT1A3, UGT2B7. } \\
\text { Inhibitor: } H M G C R \\
\text { Transporter genes: } A B C B 1, A B C G 2, S L C O 1 B 1, S L C O 2 B 1 \\
\text { Pleiotropic genes: } P P A R G, V C A M 1\end{array}$ \\
\hline & $\begin{array}{l}\text { Name: PRAVASTATIN SODIUM } \\
\text { Molecular Formula: } \mathrm{C}_{23} \mathrm{H}_{35} \mathrm{NaO}_{7} \\
\text { Molecular Weight: } 446.509569 \mathrm{~g} / \mathrm{mol} \\
\text { Mechanism: A competitive inhibitor of } \\
\text { 3-hydroxy-3-methylglutaryl coenzyme A } \\
\text { (HMG-CoA) reductase. } \\
\text { Effect: Anticholesteremic Agent; HMG-CoA Reductase } \\
\text { Inhibition; Heart-health effects; Immune response } \\
\text { modulation; MHC II suppression. }\end{array}$ & $\begin{array}{l}\text { Pathogenic genes: } A B C A 1, A C E, A P O A 1, A P O A 5, A P O B, A P O C 3, A P O E, C E T P \text {, } \\
C Y P 7 A 1, F G B, L D L R, L I P C, L P L, N O S 3 \\
\text { Mechanistic genes: } A P O A 1, A P O B, A P O C 3, A P O E, C R P, H M G C R, L D L R, I L 1 B, I L 6 \text {, } \\
\text { IL10, MMP2, NOS3 } \\
\text { Metabolic genes: } \\
\text { Substrate: } A B C B 1, A B C C 2, C Y P 3 A 4 \text { (minor), SLCO1B1, SLCO2B1, UGT1A3 } \\
\text { Inhibitor: } A B C B 1, C Y P 2 C 8, C Y P 2 C 9 \text { (weak), CYP2C19 (weak), CYP2D6 (weak), } \\
\text { CYP3A4 (weak), HMGCR } \\
\text { Inducer: CYP1A1, CYP2B6, CYP2E1 } \\
\text { Transporter genes: ABCA1, ABCB1, ABCB11. ABCG2, SLC22A8, SLCO1A2, SLCO1B1, } \\
\text { SLCO1B3, SLCO2B1 } \\
\text { Pleiotropic genes: ACE, ALDH1A1, APOE, CBS, FGB, HTR3B, IL6, IL10, ITGB3, LEP, } \\
\text { MTHFR, MMP2, MMP3, NOS3, TP53, USP5 }\end{array}$ \\
\hline & $\begin{array}{l}\text { Name: ROSUVASTATIN CALCIUM } \\
\text { Molecular Formula: } \mathrm{C}_{44} \mathrm{H}_{54} \mathrm{CaF}_{2} \mathrm{~N}_{6} \mathrm{O}_{12} \mathrm{~S}_{2} \\
\text { Molecular Weight: } 1001.137366 \mathrm{~g} / \mathrm{mol} \\
\text { Mechanism: Inhibitor of HMG-CoA reductase, } \\
\text { rate-limiting enzyme in cholesterol synthesis. This } \\
\text { results in compensatory increase in expression of LDL } \\
\text { receptors on hepatocyte membranes and stimulation of } \\
\text { LDL catabolism. } \\
\text { Effect: Anticholesteremic Agent; HMG-CoA } \\
\text { Reductase Inhibition; Heart-health effects; } \\
\text { Antineoplastic activity. }\end{array}$ & $\begin{array}{l}\text { Pathogenic genes: } A B C A 1, A C E, A P O A 1, A P O A 5, A P O B, A P O C 3, A P O E, C E T P \\
C Y P 7 A 1, F G B, L D L R, L I P C, L P L, N O S 3 \\
\text { Mechanistic genes: } A P O A 1, A P O B, C E T P, F G B, H M G C R, L D L R, L P L, N O S 3 \\
\text { Metabolic genes: } \\
\text { Substrate: } A B C B 1, A B C C 1, A B C C 4, A B C G 2, C Y P 2 C 9 \text { (minor), CYP2C19, CYP3A4 } \\
\text { (minor), SLC10A1, SLCO1A2, SLCO1B1, SLCO1B3, SLCO2B1, UGT1A3 } \\
\text { Inhibitor: CYP3A4,CYP3A5, HMGCR, SLCO1B1 } \\
\text { Inducer: CYP2B6, CYP2C8,CYP2C9,CYP2C19, CYP3A4 } \\
\text { Transporter genes: } A B C A 1, A B C B 1, A B C B 11, A B C G 2, S L C 10 A 1, S L C 22 A 8, S L C O 1 A 2 \text {, } \\
\text { SLCO1B1, SLCO1B3, SLCO2B1 } \\
\text { Pleiotropic genes: } A C E, A P O E, F G B, I T G B 3, N O S 3, T C F 20, U S P 5\end{array}$ \\
\hline
\end{tabular}


Table 1. Cont.

\begin{tabular}{|c|c|c|}
\hline \multicolumn{3}{|c|}{ HMG CoA Reductase Inhibitors } \\
\hline Drug & Properties & Pharmacogenetics \\
\hline & $\begin{array}{l}\text { Name: SIMVASTATIN } \\
\text { Molecular Formula: } \mathrm{C}_{25} \mathrm{H}_{38} \mathrm{O}_{5} \\
\text { Molecular Weight: } 418.56622 \mathrm{~g} / \mathrm{mol} \\
\text { Mechanism: Prodrug requiring hydrolysis in vivo for } \\
\text { activity. Inhibits HMG-CoA reductase, causing } \\
\text { subsequent reduction in hepatic cholesterol synthesis. } \\
\text { Reduces serum concentrations of total cholesterol, } \\
\text { LDL-C, Apo B, and triglycerides } \\
\text { Effect: Anticholesteremic Agent; HMG-CoA } \\
\text { Reductase Inhibition; Heart-health effects; } \\
\text { Anti-inflammatory activity }\end{array}$ & $\begin{array}{l}\text { Pathogenic genes: } A B C A 1, A P O A 1, A P O A 5, A P O B, A P O C 3, A P O E, C E T P, C Y P 7 A 1 \text {, } \\
\text { FGB, GNB3, LIPC, LDLR, LPL, NOS3 } \\
\text { Mechanistic genes: } A B C A 1, A P O A 1, A P O B, A P O E, C E T P, H M G C R, I L 6, L D L R, \\
L P L, V C A M 1 \\
\text { Metabolic genes: } \\
\text { Substrate: } A B C B 1, C Y P 2 C 8 \text { (minor), CYP2C9, (minor), CYP2C19 (minor), CYP2D6, } \\
\text { CYP3A4 (major), CYP3A5 ,POR, SLCO1B1, UGT1A3 } \\
\text { Inhibitor: CYP2C8 (weak), CYP2C9 (weak), CYP2C19 (strong), CYP2D6 (weak), } \\
\text { CYP3A4 (moderate), HMGCR } \\
\text { Inducer: CYP2B6 } \\
\text { Transporter genes: } A B C A 1, A B C B 1, A B C B 11, A B C C 2, A B C C 3, A B C G 2 \text {, } \\
\text { SLCO1B1, SLCO1B3 } \\
\text { Pleiotropic genes: } A P O E, F 2, F G B, G N B 3, N O S 3, P R N P, T N F, V C A M 1, U S P 5\end{array}$ \\
\hline
\end{tabular}

$A B C A 1$ : ATP binding cassette subfamily A member 1; $A B C B 1$ : ATP binding cassette subfamily B member 1; $A B C B 11$ : ATP binding cassette subfamily B member 11; $A B C C 1$ : ATP binding cassette subfamily $C$ member 1; $A B C C 2$ : ATP binding cassette subfamily $C$ member 2; $A B C C 3$ : ATP binding cassette subfamily C member 3; $A B C C 4$ : ATP binding cassette subfamily C member 4; $A B C G 2$ : ATP binding cassette subfamily G member 2; $A C E$ : angiotensin I converting enzyme; $A L D H 1 A 1$ : aldehyde dehydrogenase 1 family member A1; $A P O A 1$ : apolipoprotein A-I; APOA5: apolipoprotein A-V; APOB: apolipoprotein B; APOC3: apolipoprotein C-III; APOE: apolipoprotein E; CBS: cystathionine-beta-synthase; CETP: cholesteryl ester transfer protein, plasma; CRP: C-reactive protein, pentraxin-related; CYP1A1: cytochrome P450 family 1 subfamily A member 1; CYP1A2: cytochrome P450 family 1 subfamily A member 2; CYP2B6: cytochrome P450 family 2 subfamily B member 6; CYP2C19: cytochrome P450 family 2 subfamily C member 19; CYP2C8: cytochrome P450 family 2 subfamily C member 8; CYP2C9: cytochrome P450 family 2 subfamily C member 9; CYP2D6: cytochrome P450 family 2 subfamily D member 6; CYP2E1: cytochrome P450 family 2 subfamily E member 1; CYP3A4: cytochrome P450 family 3 subfamily A member 4; CYP3A5: cytochrome P450 family 3 subfamily A member 4; CYP7A1: cytochrome P450 family 7 subfamily A member 1; CYP11B2: cytochrome P450 family 11 subfamily B member 2; ESR1: estrogen receptor 1; F2: coagulation factor II, thrombin; FGB: fibrinogen beta chain; GNB3: guanine nucleotide binding protein (G protein), beta polypeptide 3; HMG-CoA: 3-hydroxy-3-methylglutaryl coenzyme A; HMGCR: 3-hydroxy-3-methylglutaryl-CoA reductase; HTR3B: 5-hydroxytryptamine (serotonin) receptor 3B, ionotropic; IL1B: interleukin 1 beta; IL6: interleukin 6; IL10: interleukin 10; ITGB3: integrin subunit beta 3; KCNH2: potassium voltage-gated channel subfamily H member 2; LDLR: low density lipoprotein receptor; LEP: leptin; LIPC: lipase C, hepatic; LPL: lipoprotein lipase; MMP2: matrix metallopeptidase 2; MMP3: matrix metallopeptidase 3; MTHFR: methylenetetrahydrofolate reductase $(\mathrm{NAD}(\mathrm{P}) \mathrm{H})$; MTTP: microsomal triglyceride transfer protein; NOS3: nitric oxide synthase 3; NR1I2: nuclear receptor subfamily 1 group I member 2; NR1I3: nuclear receptor subfamily 1 group I member 3; PON1: paraoxonase 1; POR: P450 (cytochrome) oxidoreductase; PPARD: peroxisome proliferator activated receptor delta; PPARG: peroxisome proliferator activated receptor gamma; PRNP: prion protein; SLC10A1: solute carrier family 10 (sodium/bile acid cotransporter), member 1; SLC15A1: solute carrier family 15 (oligopeptide transporter), member 1; SLC22A8: solute carrier family 22 (organic anion transporter), member 8; SLCO1A2: solute carrier organic anion transporter family member 1A2; SLCO1B1: solute carrier organic anion transporter family member 1B1; SLCO1B3: solute carrier organic anion transporter family member 1B3; SLCO2B1: solute carrier organic anion transporter family member 2B1; TCF20: transcription factor 20 (AR1); TNF: tumor necrosis factor; TP53: tumor protein p53; UGT1A3: UDP glucuronosyltransferase 1 family, polypeptide A3; UGT2B7: UDP glucuronosyltransferase 2 family, polypeptide B7; USP5: ubiquitin specific peptidase 5 (isopeptidase T); VCAM1: vascular cell adhesion molecule 1. 


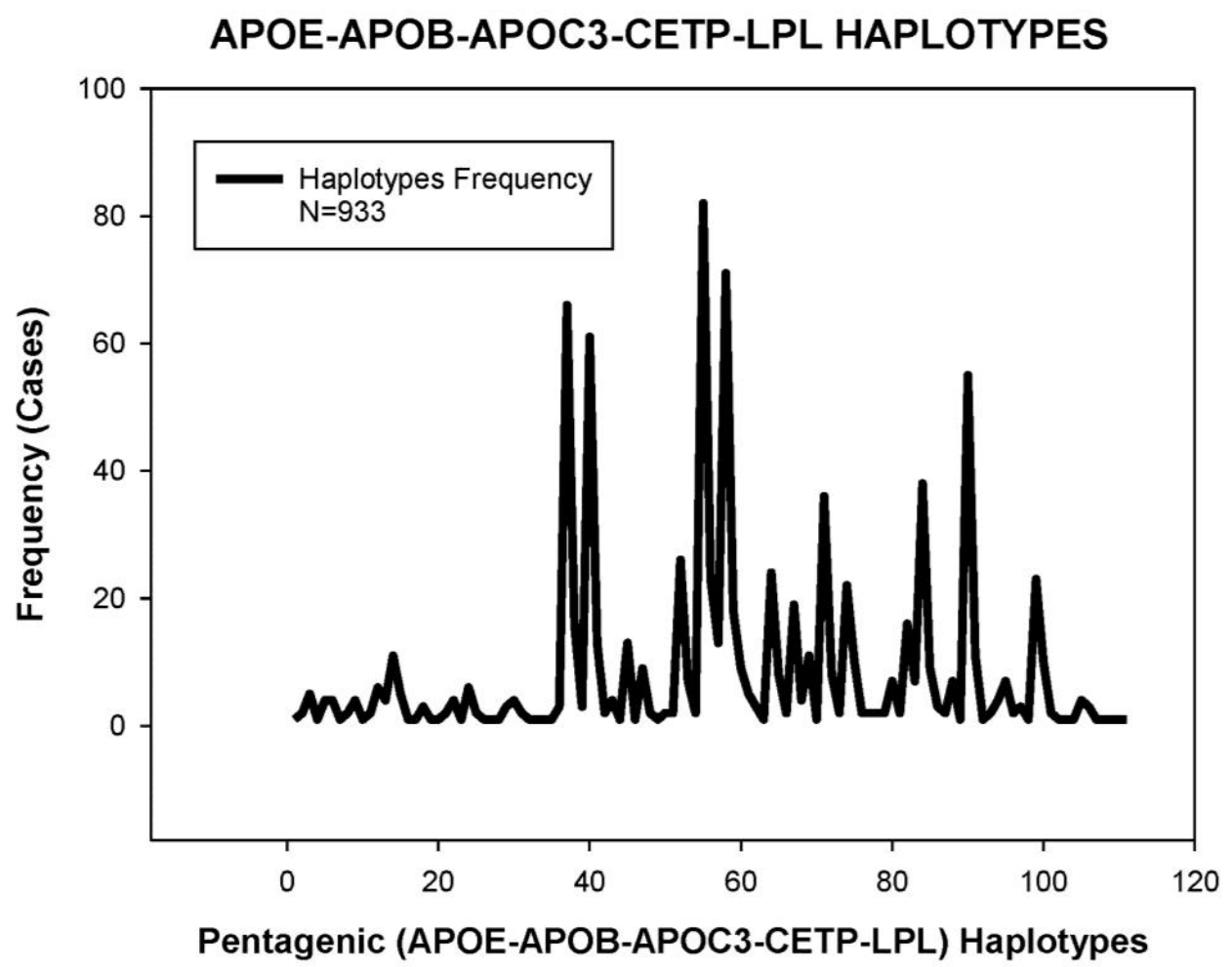

Figure 5. Distribution and frequency of pentagenic haplotypes integrating $A P O E, A P O B, A P O C 3$, CETP, and LPL genotypes in patients with Alzheimer's disease.

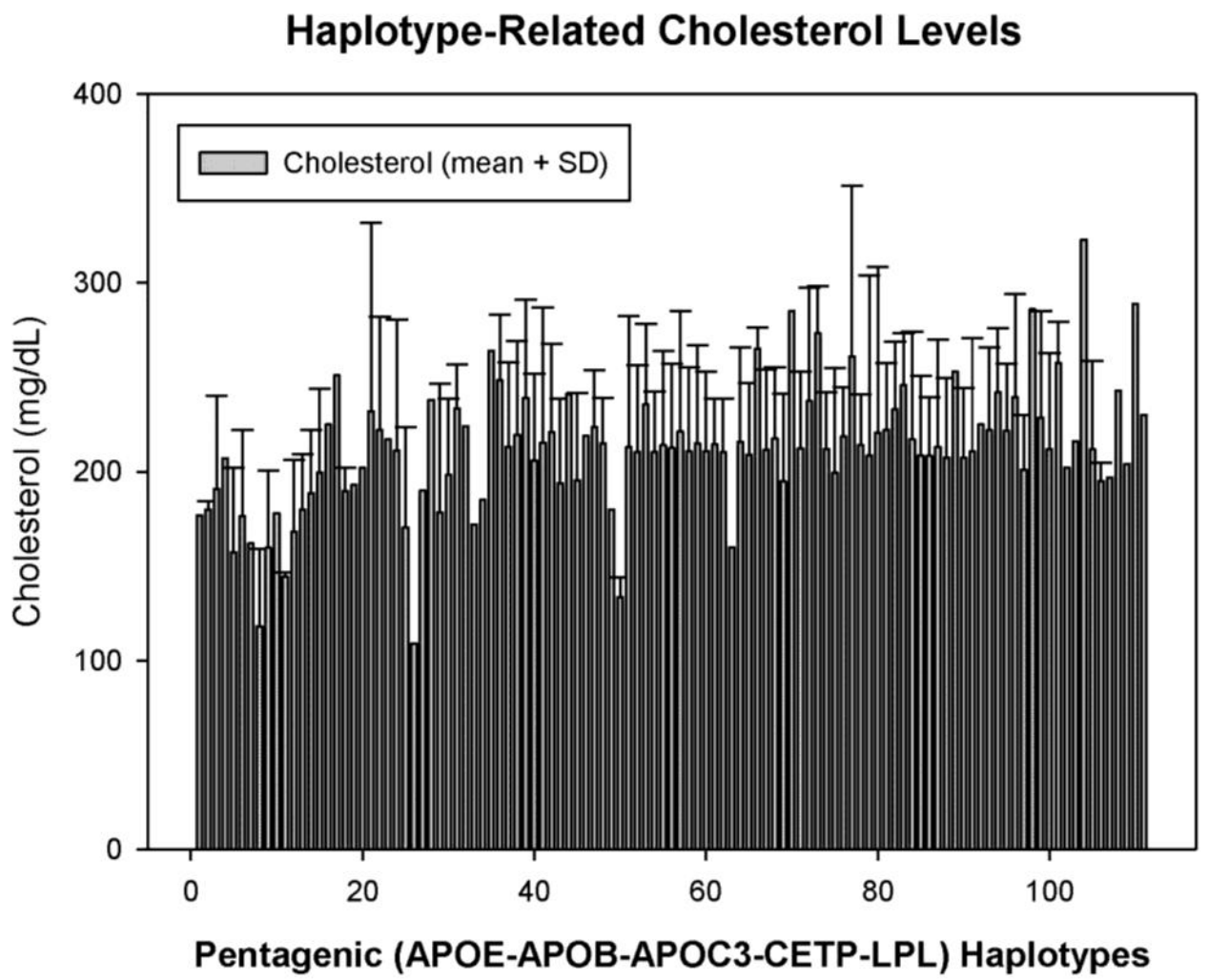

Figure 6. Pentagenic haplotype-related basal cholesterol levels in patients with Alzheimer's disease. 
The results of $A P O E$-related cholesterol response to hypolipemic treatment in hypercholesterolemic $\mathrm{AD}$ patients revealed that in absolute terms all APOE variants respond similarly (RR $>70 \%$ ) to treatment, with a significant reduction in CHO levels $(p<0.001)$ (Figure 7); however, genotype-related correlation analysis case by case (Figure 8 ) and comparative correlation analyses of APOE variants show a clear differential APOE-related pattern of $\mathrm{CHO}$ response to treatment [17].

\section{APOE-Related Cholesterol Response to Hypolipemic Treatment} Alzheimer's Disease

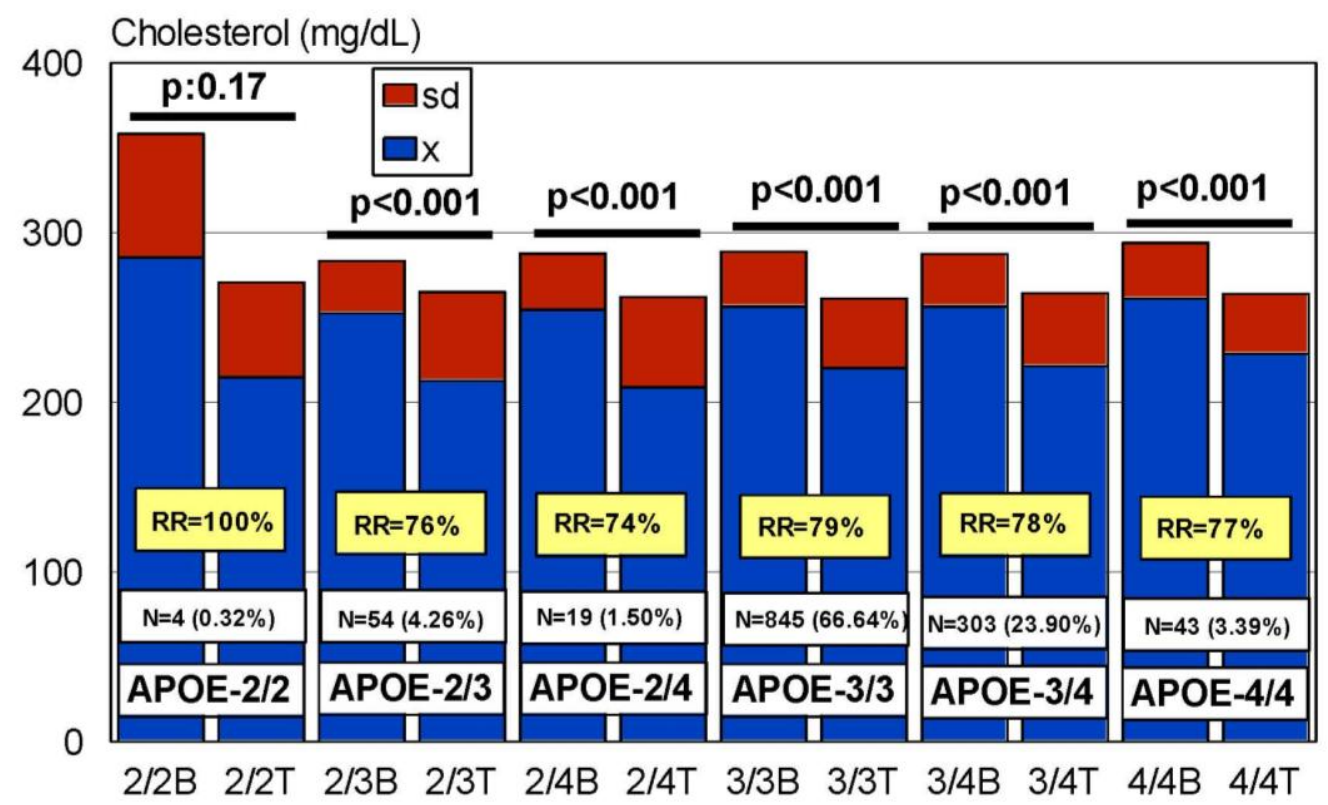

Figure 7. APOE-related cholesterol response to a hypolipemic treatment in hypercholesterolemic patients with Alzheimer's disease. B: Basal values; T: Treatment (Atorvastatin: 10-20 mg/day; LipoEsar: $500 \mathrm{mg}$ /day).

Carriers of $A P O B-C / C, A P O B-C / T$, and $A P O B-T / T$ variants exhibit a similar response (RR $>80 \%$ ), with a significant decrease in $\mathrm{CHO}$ levels after treatment (Figure 9) and almost identical efficiency in comparative analyses. APOC3-C/C, APOC3-C/G, and APOC3-G/G carriers also respond similarly $(p<0.001)$ (RR $>80 \%)$ (Figure 10), with a differential comparative profile among APOC3 variants. CETP-A/A, CETP-A/G, and CETP-G/G carriers show an identical response $(p<0.001 ; \mathrm{RR}>80 \%)$ (Figure 11), with insignificant variability in comparative studies among CETP variants. The same therapeutic response is observed in $L P L-C / C, L P L-C / G$, and $L P L-G / G$ carriers $(p<0.001 ; R R>80 \%)$ (Figure 12); however, in this case, $L P L-\mathrm{C} / \mathrm{C}$ are the best responders, $L P L-\mathrm{C} / \mathrm{G}$ are intermediate responders, and $L P L-G / G$ are the most heterogeneous responders [17]. 

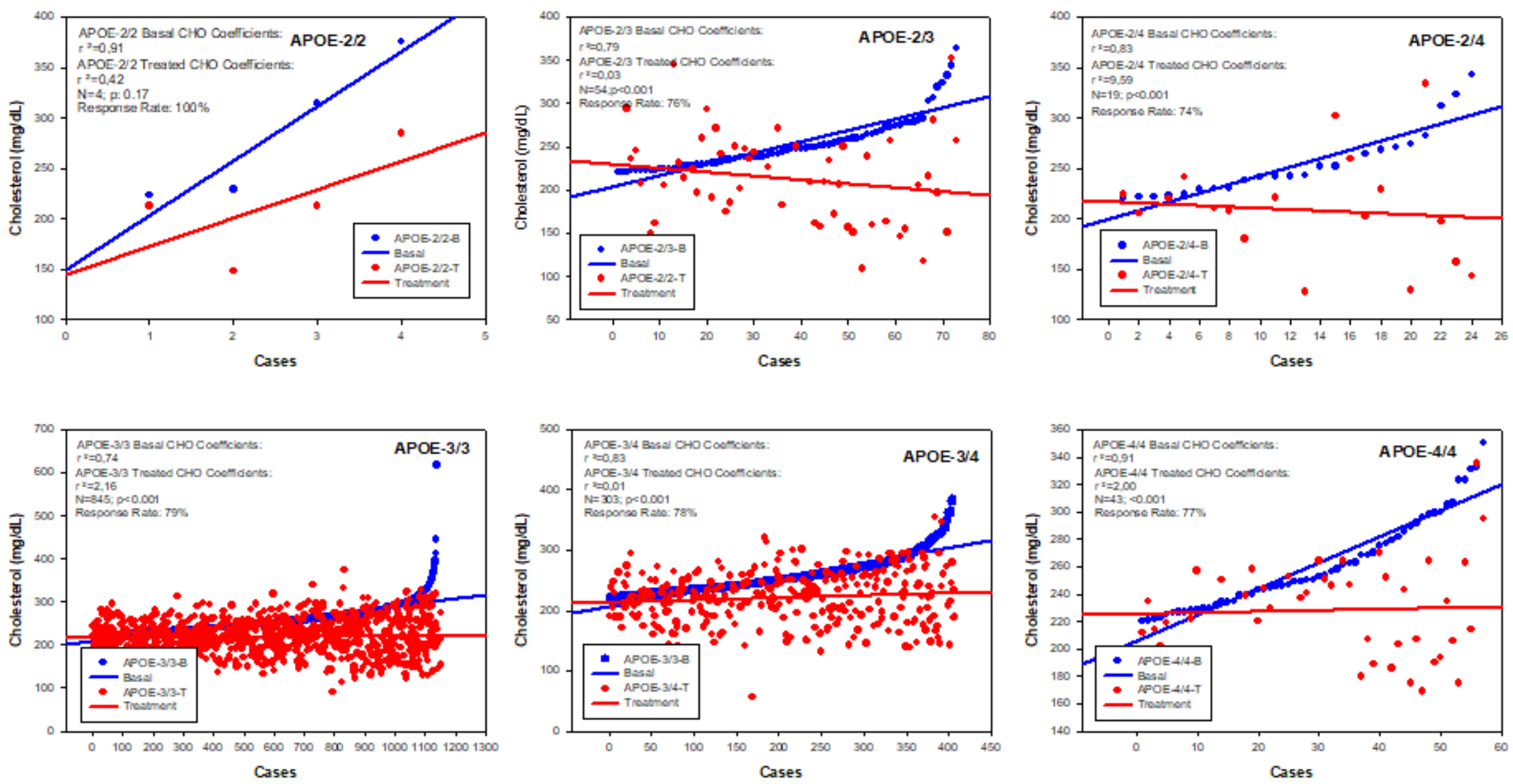

Figure 8. APOE-related individual response of cholesterol to a hypolipemic treatment (Atorvastatin + LipoEsar) in hypercholesterolemic patients with Alzheimer's disease. 


\section{APOB-Related Cholesterol Response to Hypolipemic Treatment}

Alzheimer's Disease

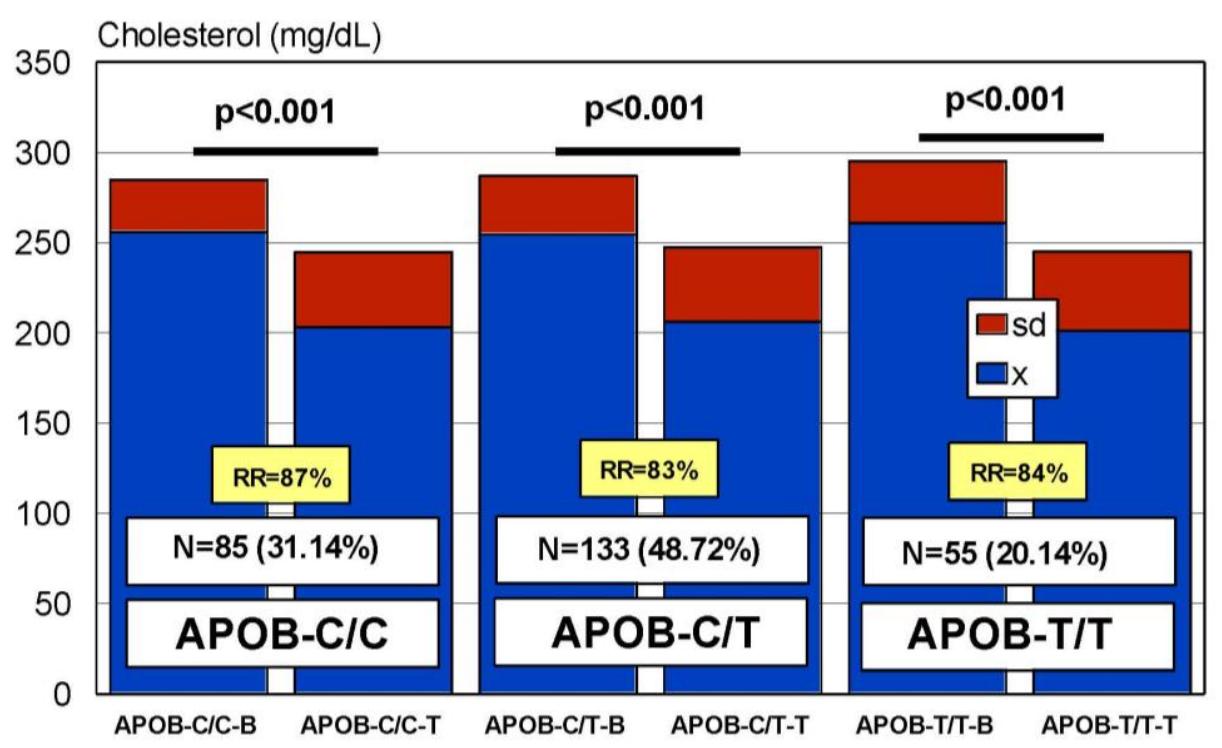

Figure 9. $A P O B$-related cholesterol response to a hypolipemic treatment in hypercholesterolemic patients with Alzheimer's disease (Atorvastatin: 10-20 mg/day; LipoEsar: 500 mg/day).

\section{APOC3-Related Cholesterol Response to Hypolipemic Treatment Alzheimer's Disease}

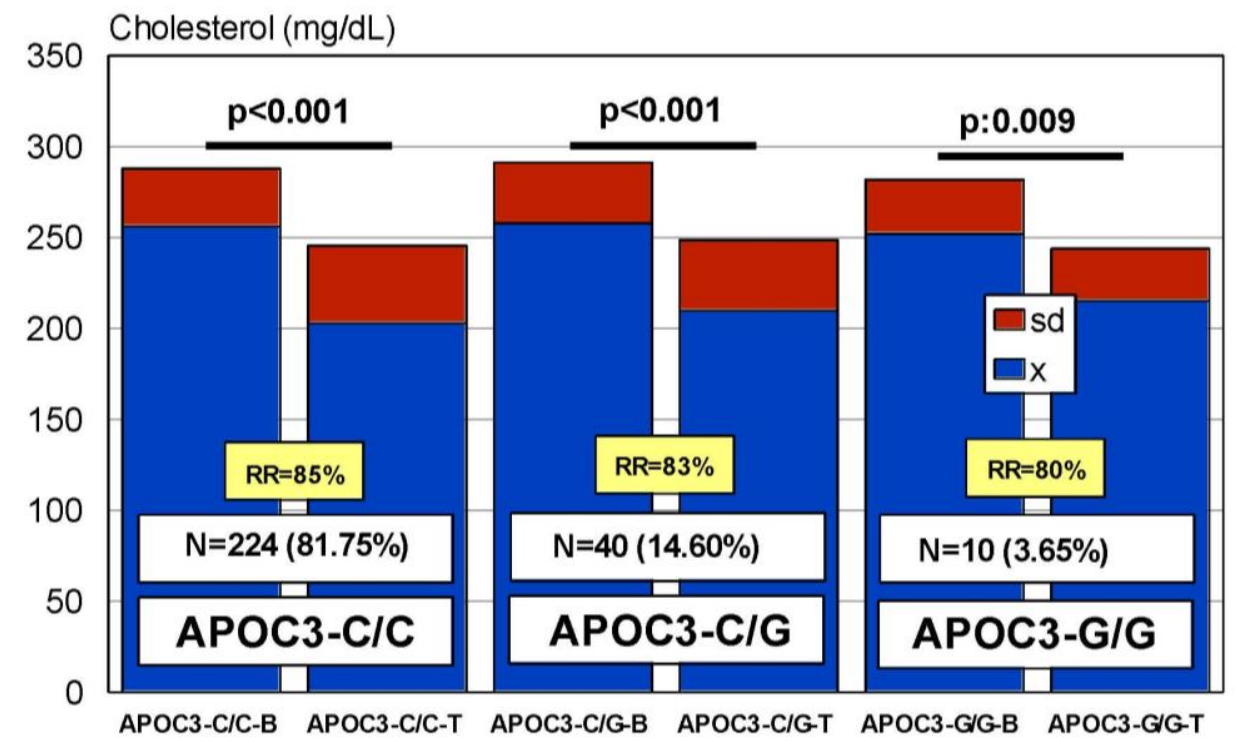

Figure 10. APOC3-related cholesterol response to a hypolipemic treatment in hypercholesterolemic patients with Alzheimer's disease (Atorvastatin: 10-20 mg/day; LipoEsar: 500 mg/day). 


\section{CETP-Related Cholesterol Response to Hypolipemic Treatment}

\section{Alzheimer's Disease}



Figure 11. CETP-related cholesterol response to a hypolipemic treatment in hypercholesterolemic patients with Alzheimer's disease (Atorvastatin: 10-20 mg/day; LipoEsar: 500 mg/day).

\section{LPL-Related Cholesterol Response to Hypolipemic Treatment}

\section{Alzheimer's Disease}

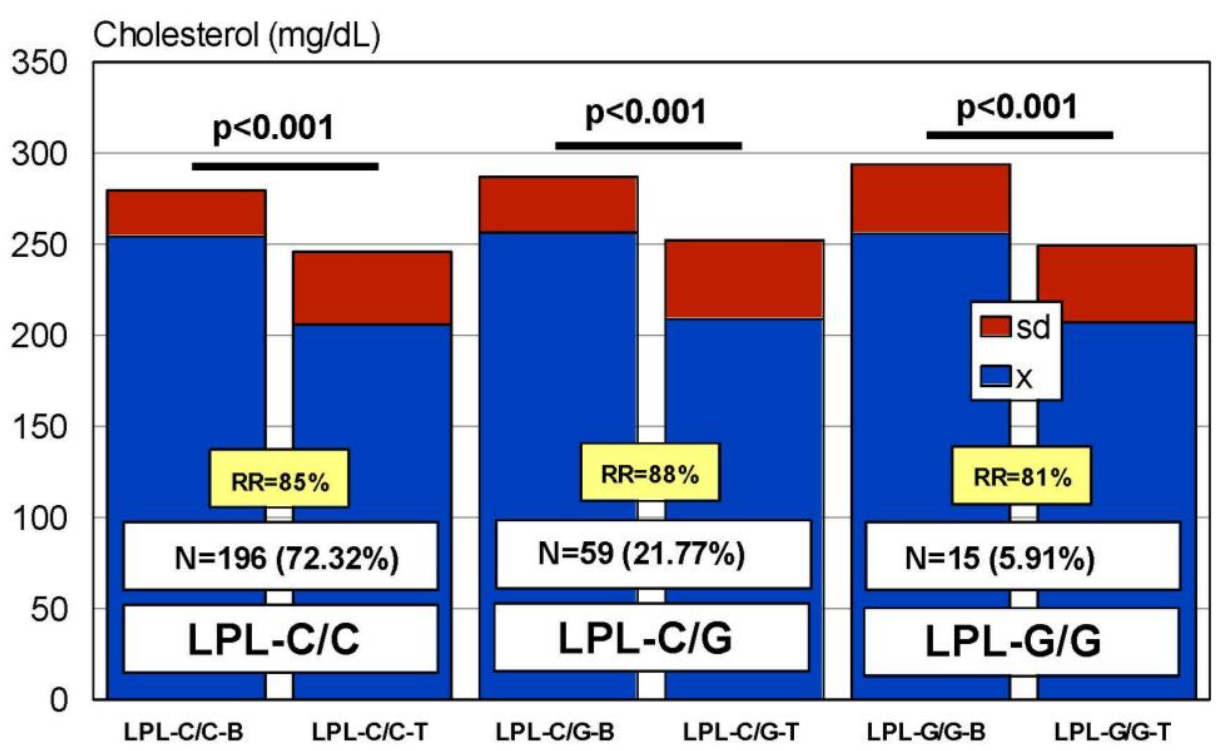

Figure 12. $L P L$-related cholesterol response to a hypolipemic treatment in hypercholesterolemic patients with Alzheimer's disease (Atorvastatin: 10-20 mg/day; LipoEsar: 500 mg/day).

CYP haplotype-related blood total CHO levels are very heterogeneous, but absolute values of total $\mathrm{CHO}$ among the most frequent haplotypes are almost identical. The histograms of frequency associated with $\mathrm{CHO}$ levels are qualitatively different among carriers of different $C Y P$ variants. Basal $\mathrm{CHO}$ levels are higher in $\mathrm{AD}$ patients harboring the $C Y P 2 D 6-{ }^{*} 1 /{ }^{*} 1$ and ${ }^{*} 1 x N /{ }^{*} 1$ genotypes than in the corresponding GP genotypes, but no differences have been found according to the EM, IM, PM, or UM condition. The therapeutic response according to SNPs of metabolic genes (CYP2D6, CYP2C9, CYP2C19, 
and CYP3A4/4) in hypercholesterolemic patients is variable and geno-phenotype-dependent. Although all $C Y P 2 D 6$ variants exhibit a positive response to treatment, significant differences have only been detected in $2 D 6-{ }^{*} 1 /{ }^{*} 1,2 D 6-{ }^{*} 1 /{ }^{*} 4$, and $2 D 6-{ }^{*} 1{ }^{*} 6$ carriers. In absolute values, CYP2D6 extensive, intermediate, poor, and ultra-rapid metabolizers behave in a similar manner with a significant reduction in CHO levels; however, the RR is different in EMs (81\%), IMs (78\%), PMs (84\%), and UMs $(90 \%)$, indicating a variable efficiency of CYP2D6 enzymes. The comparative analysis indicates that carriers of mutant enzymes (PMs > UMs), with limitations in drug metabolism, display a more efficient response to hypolipemic treatment [17]. No differences are present in basal $\mathrm{CHO}$ levels between the GP and AD patients related to CYP2C9 genotypes. CYP2C9-EMs, -IMs, and -PMs show a similar response, with lower RR (75\%) in PMs as compared with EMs (81\%) and IMs (82\%), and a clear differential comparative profile. AD cases harboring the $C Y P 2 C 19-{ }^{*} 1 /{ }^{*} 2$ genotype, corresponding to CYP2C19-IMs, exhibit higher basal CHO levels than their homologs in the GP. The CHO response among CYP2C19-EMs, IMs, PMs, and UMs is more variable, with PMs showing a deficient response in comparison to EMs, IMs, and UMs, and a clearly different behavioral profile, especially in PMs and UMs [17]. CYP3A4/5 geno-phenotypes in AD and GP show similar basal CHO levels. CYP3A4/5-RMs respond poorly to hypolipemic treatment, with the worst RR (66\%), whereas CYP3A4/5-EMs and -IMs exhibit an excellent response $(p<0.001 ; \mathrm{RR}>80 \%)$ [17].

Most of these effects can, in part, be explained on a pharmacogenetic basis. It is obvious that a simple stratification of patients according to single genotypes is of poor value for a fine interpretation of pharmacogenetic results; however, the integration of gene clusters associated with specific phenotypes yields informative haplotypes with potential utility in pharmacogenetic studies. It is likely that thousands of genes are involved in $\mathrm{CHO}$ metabolism, and probably not a single gene plays an absolute dominant role over the others; however, some genes exert a powerful effect on other congeners associated with a specific pathogenic cascade (e.g., APOE in AD) or a pharmacogenetic pathway (e.g., $A P O E$ vs. $C Y P s$ in AD treatment with donepezil) $[3,10,25,36,37]$. The lipid-lowering effects and the anti-atherosclerotic properties of LipoEsar are $A P O E$-dependent, with APOE-3 carriers acting as the best responders and $A P O E-4$ carriers behaving as the worst responders [10,58].

Statins (--Hydroxy-3-Methylglutaryl-Coenzyme A Reductase (HMGCR) inhibitors) are among the most prescribed drugs worldwide (Table 1). Inhibition of HMGCR results in decreased intrahepatic CHO synthesis, together with the upregulation of LDL-CHO receptors, increased LDL-CHO uptake by hepatocytes, and decreased levels of systemic LDL-CHO. The most relevant ADRs of statins are muscle, liver, and brain toxicity.

Several pathogenic (ACE, APOA1, APOA5, APOB, APOC3, APOE, CETP, FGB, GNB3, LIPC, MMP3, MTTP, NOS3, PON) and mechanistic genes (ABCB1, ABCC1, APOA1, APOA5, APOB, APOC3, APOE, CRP, CYP11B2, HMGCR, IL10, IL6, LDLR, MMP3, PON1, TNF) influence the effects of atorvastatin. This statin is a major substrate of CYP2C8 and CYP3A4/5; it is a strong inhibitor of CYP2C19, a moderate inhibitor of ABCB1, CYP2B6, CYP2C8, CYP2C9, CYP2C19, CYP2D6, CYP3A4, and HMGCR, and an inducer of CYP2B6 and CYP7A1. Atorvastatin is transported by ABCA1, ABCB1, ABCB11, $\mathrm{ABCC} 1, \mathrm{ABCC} 2, \mathrm{ABCC} 3, \mathrm{ABCG} 2, \mathrm{SLCO1B} 1$, and SLCO1B3 proteins and interacts with the products of various pleiotropic genes (APOA1, APOE, CRP, CYP11B2, ESR1, GNB3, HTR3B, IL6, IL10, ITGB3, $M M P 3, T N F$, and USP5) [16,36].

HMGCR variants (rs17244841, rs3846662, and rs17238540) (H7 haplotype) are responsible for an attenuated lipid-lowering response to statins $[59,60]$. Sex-related changes in cholesterol response to statins have been reported in carriers of the HMGCR-AA genotype at rs3846662, who have higher levels of total and LDL-cholesterol. The percentage reduction in LDL-cholesterol upon statin treatment is decreased in women with the AA genotype compared with women without it. In hypercholesterolemic patients, HMGCR alternative splicing may explain $22-55 \%$ of the variance in statin response [61].

Several polymorphisms in the SLCO1B1 gene may alter transport of statins into the liver. The SLCO1B1 521C (rs4149056) variant is associated with diminished effects of simvastatin, atorvastatin, lovastatin, and pravastatin [62]. Another transporter that modifies the effects of statins is $A B C B 1$, 
especially in carriers of the ABCB1-1236T (rs1128503), 2677T (rs2032582), and 3435T (rs1045642) variants (TTT haplotype) [63]. Other transporters potentially affecting statin transport and metabolism include ABCC2, ABCG2, ABCB11, SLC15A1, SLC22A6, SLC22A8, SLCO2B1, SCLO1B3, and SLCO1B3 [59]. Statin metabolism is influenced by the following enzymes: CYP3A4/5, CYP2C8/9, CYP2C19, CYP2D6, UGT1A1, UGT1A3, and UGT2B7 [59]. The deficient CYP3A4 enzyme in CYP3A4*22 (rs35599367) carriers alters the pharmacokinetics and pharmacodynamics of simvastatin, atorvastatin, and lovastatin [64], and the CYP3A5*3 (rs776746) variant (loss-of-function allele) causes a high increase in the bioavailability of simvastatin $[65,66]$. The powerful effect of atorvastatin in CYP3A4/5-IMs is the result of a poor metabolization of atorvastatin by mutant CYP3A4/5 enzymes, since atorvastatin is a major substrate of CYP3A4/5. In contrast, the lack of effect in CYP3A4/5-RMs results from a rapid destruction of the drug in the liver mediated by excessive CYP3A4/5 enzymatic activity. Therefore, the dose of statins should be adjusted to the metabolizing condition of each patient to optimize the lipid-lowering effects of statins and to avoid toxicity $[36,64]$. Furthermore, the co-administration of the nutraceutical LipoEsar enhances the hypolipemic effect of atorvastatin and facilitates a dose reduction of the statin by $50 \%$, minimizing potential ADRs in susceptible patients $[10,16,17]$.

\section{Pharmacogenetics of Hypertension in Alzheimer's Disease}

Hypertension is considered a modifiable risk factor for $\mathrm{AD}$, together with diabetes, obesity, physical inactivity, depression, smoking, and low educational attainment [67]. Hypertension is also associated with the worsening of cognitive function, but not with evidence of increased amyloidopathy and/or tauopathy [68]. However, in AD, both hypotension and hypertension may induce deleterious effects on brain function, cognition, and psychomotor praxis. In a sample of 1308 healthy subjects (age: $55.27 \pm 10.82$ years; range: 40-94; Females: 607; Males: 701) and 1929 AD cases (age: $67.59 \pm 11.76$ years; range: $40-98$; Females: 1175; Males: 754), we found that hypertension is an age-dependent condition (Figure 13). Systolic blood pressure (SBP) in AD (139.44 $\pm 21.79 \mathrm{~mm} \mathrm{Hg}$ ) tends to be higher than in the control population with no family history of dementia (132.41 $\pm 21.14 \mathrm{~mm} \mathrm{Hg})$ $(p<0.001)$; in contrast, diastolic blood pressure (DBP) does not show differences between both groups. Among AD cases, we found $15.81 \%$ of patients with SBP < $120 \mathrm{~mm} \mathrm{Hg} ; 57.60 \%$ were normotensive (SBP: $120-150 \mathrm{~mm} \mathrm{Hg}$ ), and 26.59\% were hypertensive (SBP > $150 \mathrm{~mm} \mathrm{Hg}$ ). Regarding DBP, $9.80 \%$ were hypotensive (DBP < $70 \mathrm{~mm} \mathrm{Hg}$ ), $65.47 \%$ normotensive (DBP: $70-85 \mathrm{~mm} \mathrm{Hg}$ ), and $24.73 \%$ hypertensive (DBP > $85 \mathrm{~mm} \mathrm{Hg}$ ). In the control population, $14.67 \%$ showed systolic hypertension and $17.30 \%$ diastolic hypertension. Hypertension was almost $50 \%$ less frequent in the control population than in the AD cohort $(p<0.0001)$.

In general, over $20 \%$ of $\mathrm{AD}$ patients are hypertensive and receive different modalities of hypotensive agents, including central $\alpha$-adrenergic agonists, vasodilators, diuretics, nitrates, nitrites, phosphodiesterase inhibitors, calcium channel blockers, angiotensin-converting enzyme inhibitors, angiotensin II receptor antagonists, mineralcorticoid (aldosterone) receptor antagonists, renin inhibitors, and other plural miscellaneous compounds (Table 2). 


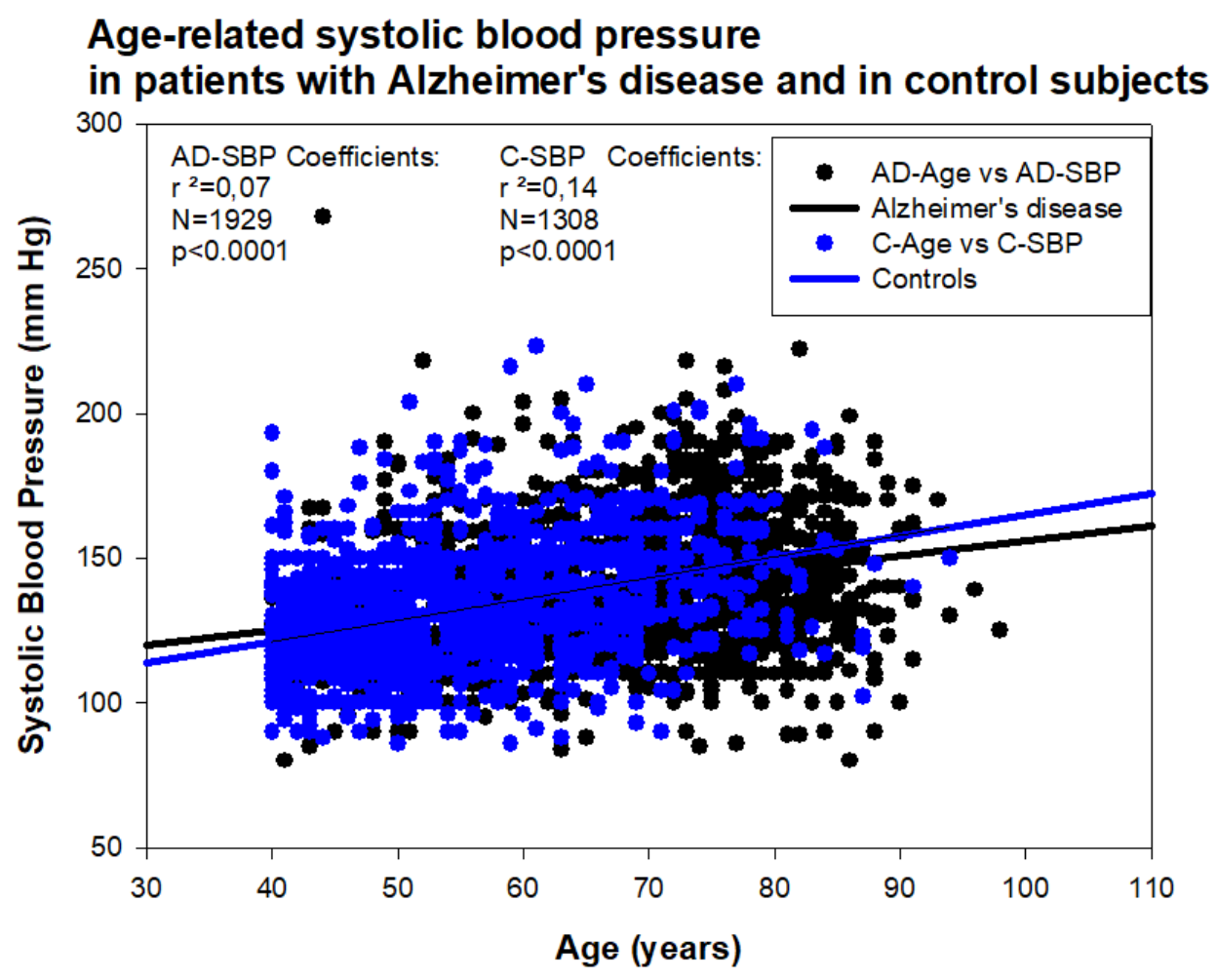

Figure 13. Age-related systolic blood pressure in patients with Alzheimer's disease and in the control population.

In our casuistic, we treated hypertensive AD patients with Enalapril (10-20 mg/day) for one month and performed a pharmacogenetic study assessing the potential influence of APOE, NOS3, $A C E, A G T$, and $C Y P 2 D 6,2 C 19,2 C 9$, and $3 A 4 / 5$ variants on blood pressure response to this competitive inhibitor of the angiotensin-converting enzyme. In AD patients, SBP decreased from $139.44 \pm 21.79$ to $136.28 \pm 21.13 \mathrm{~mm} \mathrm{Hg}(p<0.0001)$; and DBP decreased from $79.04 \pm 11.02$ to $77.78 \pm 10.64 \mathrm{~mm} \mathrm{Hg}$ $(p=0.004)$. A similar response was observed in hypertensive non-demented patients. Analysis of the genotype-related blood pressure response to APOE (Figure 14), NOS3 (Figure 15), ACE (Figure 16), and $A G T$ variants (Figure 17) revealed that specific polymorphisms in these genes differentially influence the hypotensive effect of Enalapril in AD patients. For instance, only APOE-3/3 and APOE-3/4 carriers responded with significant reductions in SBP $(p<0.001)$ and DBP values $(p<0.05)$ (Figure 14); and APOE-4 carriers tended to show higher hypertensive levels than APOE-4 non-carriers (Figure 14). NOS3-G/G carriers responded better than NOS3-G/T $>N O S 3-T / T$ carriers (Figure 15). Polymorphic variants of the $A C E$ rs4332 (547C $>\mathrm{T}$ ) SNP did not show any effect; however, $A C E-I / D$ carriers of the Alu $287 \mathrm{bp}$ Indel I/D exhibited a better response than $A C E-D / D$ and $A C E-I / I$ carriers in SBP, and ACE-I/I carriers responded better in DBP than $A C E-D / D$ and $A C E-I / D$ (Figure 16). Probably, the clearest response was observed among $A G T-A / A$ and $A G T-A / G$ carriers, who responded significantly better than $A G T-G / G$ carriers (Figure 17). Concerning CYP variants, CYP2D6-, CYP2C19-, and CYP2C9-EMs and -IMs are better responders that PMs or UMs. CYP3A4/5 variants did not show any effect on blood pressure changes. However, it is very likely that different CYP variants influence basal SBP and DBP values. 
Table 2. Pharmacological profile and pharmacogenetics of angiotensin-converting enzyme (ACE) inhibitors and angiotensin II receptor antagonists.

\begin{tabular}{|c|c|c|}
\hline \multicolumn{3}{|c|}{ Hypotensive Agents } \\
\hline \multicolumn{3}{|c|}{ Renin-Angiotensin-Aldosterone System Inhibitors } \\
\hline \multicolumn{3}{|c|}{ Angiotensin-Converting Enzyme Inhibitors } \\
\hline Drug & Properties & Pharmacogenetics \\
\hline & $\begin{array}{l}\text { Name: Benazepril Hydrochloride } \\
\text { IUPAC Name: } 1 \mathrm{H}-1 \text {-Benzazepine-1-acetic acid, } \\
\text { 3-[[1-(ethoxycarbonyl)-3-phenylpropyl]amino]-2,3,4,5-tetrahydro-2-oxo-, monohydrochloride, } \\
{\left[S-\left(R^{*}, R^{*}\right)\right]-} \\
\text { Molecular Formula: } \mathrm{C}_{24} \mathrm{H}_{28} \mathrm{~N}_{2} \mathrm{O}_{5} \cdot \mathrm{HCl} \\
\text { Molecular Weight: } 460.95 \mathrm{~g} / \mathrm{mol} \\
\text { Mechanism: Competitive inhibition of ACE activity, which regulates the conversion of angiotensin I } \\
\text { to angiotensin II, a potent vasoconstrictor, with resultant lower levels of angiotensin II which causes } \\
\text { an increase in plasma renin activity and a reduction in aldosterone secretion. } \\
\text { Effect: Angiotensin-Converting Enzyme Inhibition. }\end{array}$ & $\begin{array}{l}\text { Mechanistic genes: } A C E, A C E 2, A D D 1, A D R B 2, A G T \text {, } \\
\text { AGTR1, MTHFR, MTR } \\
\text { Metabolic genes } \\
\text { Substrate: CYP11B2 } \\
\text { Transporter genes: } S L C 15 A 1, S L C 15 A 2\end{array}$ \\
\hline & $\begin{array}{l}\text { Name: Captopril } \\
\text { IUPAC Name: L-Proline, } 1-[(2 S)-3 \text {-mercapto-2-methyl-1-oxopropyl]- } \\
\text { Molecular Formula: } \mathrm{C}_{9} \mathrm{H}_{15} \mathrm{NO}_{3} \mathrm{~S} \\
\text { Molecular Weight: } 217.29 \mathrm{~g} / \mathrm{mol} \\
\text { Mechanism: Competitive inhibitor of angiotensin-converting enzyme (ACE). Prevents conversion of } \\
\text { angiotensin I to angiotensin II, a potent vasoconstrictor, resulting in lower levels of angiotensin II, } \\
\text { which causes an increase in plasma renin activity and a reduction in aldosterone secretion. By } \\
\text { decreasing local angiotensin II production, ACE inhibitors may decrease vascular tone by reducing } \\
\text { direct angiotensin II-induced vasoconstriction and/or angiotensin II-induced increases in sympathetic } \\
\text { activity. In hypertensive patients, captopril reduces blood pressure by decreasing total peripheral } \\
\text { resistance with no change or an increase in heart rate, stroke volume, or cardiac output (these effects } \\
\text { are independent of pre-treatment blood pressure/cardiac output). Causes arterial and possibly venous } \\
\text { dilation. In patients with congestive heart failure, captopril decreases total peripheral resistance, } \\
\text { pulmonary vascular resistance, pulmonary capillary wedge pressure, and mean arterial and right } \\
\text { atrial pressures (cardiac index, cardiac output, stroke volume, and exercise tolerance are increased; } \\
\text { heart rate decreases or is unchanged). The drug may also cause regional redistribution of blood flow, } \\
\text { principally increasing renal blood flow (glomerular filtration rate is usually unchanged) with slight or } \\
\text { no increase in flow in the forearm or hepatic vasculature, respectively. The hypotensive effect of } \\
\text { captopril persists longer than inhibition of ACE in blood (unknown whether ACE is inhibited longer } \\
\text { in vascular endothelium than in blood). Captopril alone is apparently more effective in reducing } \\
\text { blood pressure in high or normal renin hypertension. Serum prolactin concentration has been } \\
\text { reported to increase during captopril therapy. } \\
\text { Effect: Angiotensin-Converting Enzyme Inhibition. Antihypertensive Agent. }\end{array}$ & $\begin{array}{l}\text { Mechanistic genes: } A C E, A C E 2, A G T, A L B, B D K R B 2, \\
\text { CHRNA2, LTA4H, MMP2, MMP9, NOS3, REN } \\
\text { Metabolic genes } \\
\text { Substrate: CYP2D6, CYP3A4, CYP3A5, CYP11B2 } \\
\text { Transporter genes: ABCB1, SLC15A1, SLC22A6 }\end{array}$ \\
\hline
\end{tabular}


Table 2. Cont

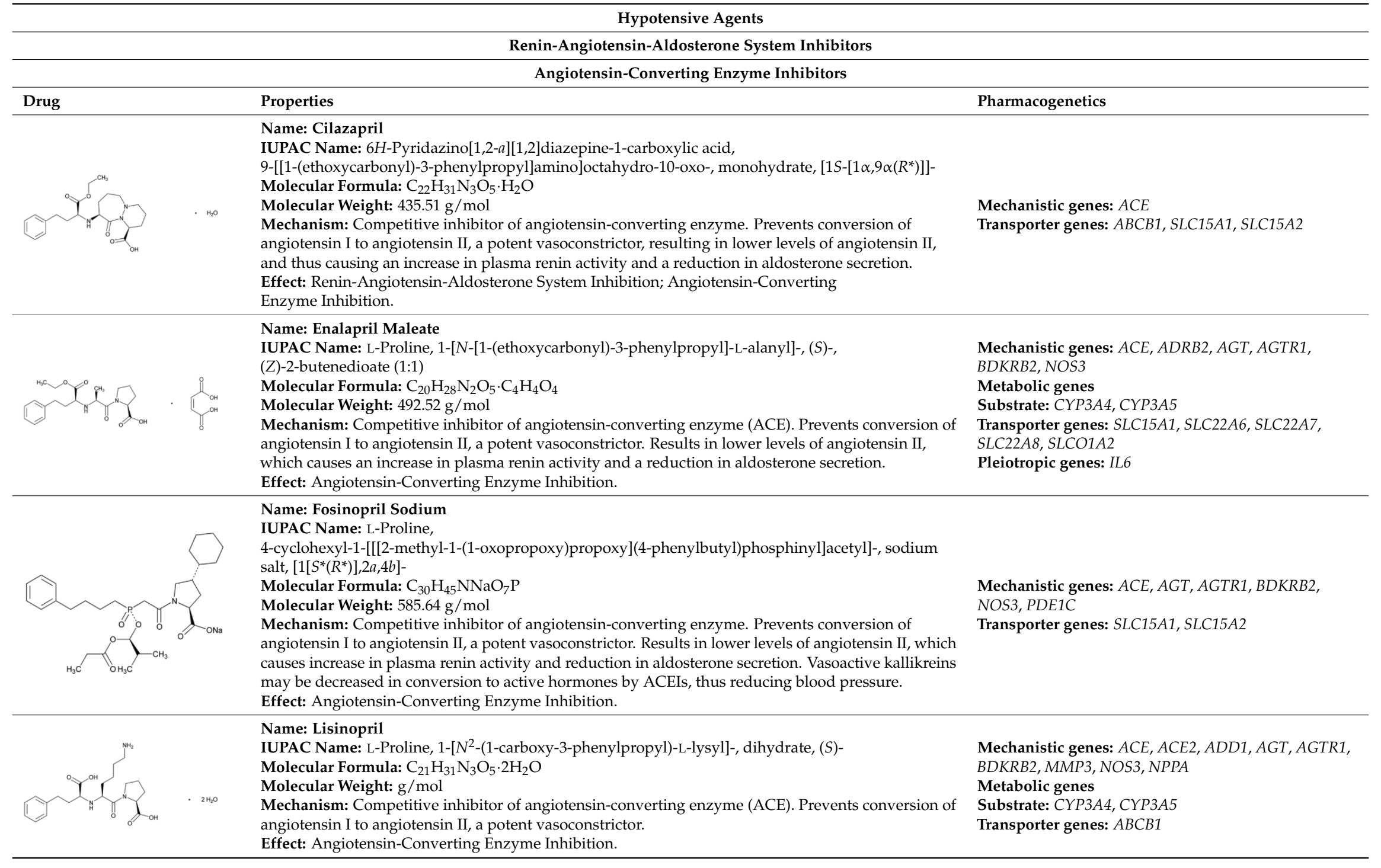


Table 2. Cont

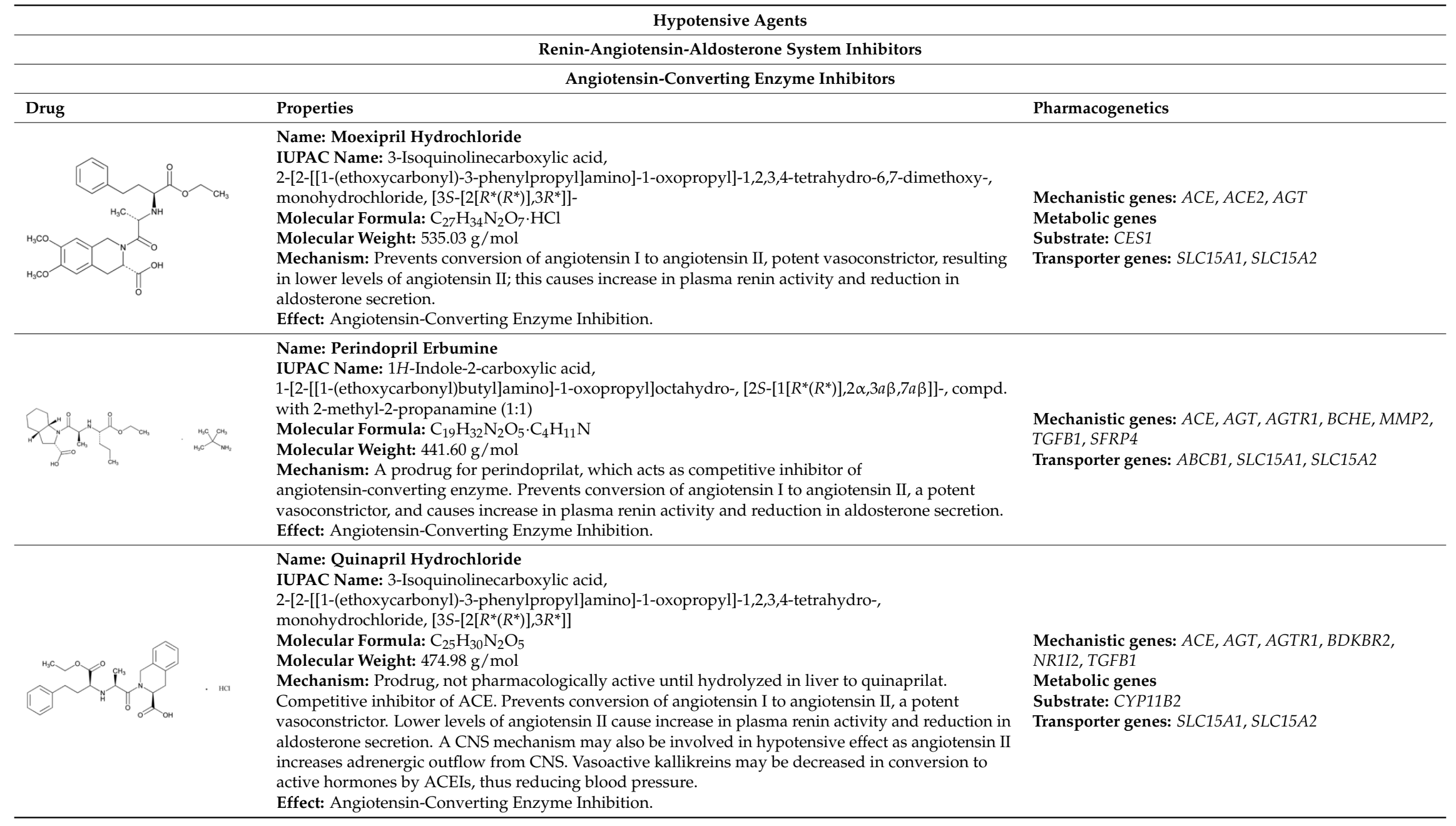


Table 2. Cont

\begin{tabular}{|c|c|c|}
\hline \multicolumn{3}{|c|}{ Hypotensive Agents } \\
\hline \multicolumn{3}{|c|}{ Renin-Angiotensin-Aldosterone System Inhibitors } \\
\hline \multicolumn{3}{|c|}{ Angiotensin-Converting Enzyme Inhibitors } \\
\hline Drug & Properties & Pharmacogenetics \\
\hline & $\begin{array}{l}\text { Name: Trandolapril } \\
\text { IUPAC Name: } \\
\text { (2S,3aR,7aS)-1-[(S)-N-[(S)-1-Carboxy-3-phenylpropyl]alanyl]hexahydro-2-indolinecarboxylic acid, } \\
\text { 1-ethyl ester } \\
\text { Molecular Formula: } \mathrm{C}_{24} \mathrm{H}_{34} \mathrm{~N}_{2} \mathrm{O}_{5} \\
\text { Molecular Weight: } 430.54 \mathrm{~g} / \mathrm{mol} \\
\text { Mechanism: Prevents formation of angiotensin II from angiotensin I. Trandolapril must undergo } \\
\text { enzymatic hydrolysis, mainly in liver, to its biologically active metabolite, trandolaprilat. A CNS } \\
\text { mechanism may also be involved in hypotensive effect as angiotensin II increases adrenergic outflow } \\
\text { from CNS. Vasoactive kallikreins may be decreased in conversion to active hormones by ACEIs, thus } \\
\text { reducing blood pressure. } \\
\text { Effect: Angiotensin-Converting Enzyme Inhibition. }\end{array}$ & $\begin{array}{l}\text { Mechanistic genes: } A C E, A D D 1 \\
\text { Metabolic genes } \\
\text { Substrate: CES1 } \\
\text { Transporter genes: } S L C 15 A 1, S L C 15 A 2\end{array}$ \\
\hline \multicolumn{3}{|c|}{ Angiotensin II Receptor Antagonists } \\
\hline Drug & Properties & Pharmacogenetics \\
\hline & $\begin{array}{l}\text { Name: Candesartan Cilexetil } \\
\text { IUPAC Name: } 1 H \text {-Benzimidazole-7-carboxylic acid, } \\
\text { 2-ethoxy-1-[[2'-(1H-tetrazol-5-yl)[1,1'-biphenyl]-4-yl]methyl]- } \\
\text { Molecular Formula: } \mathrm{C}_{24} \mathrm{H}_{20} \mathrm{~N}_{6} \mathrm{O}_{3} \\
\text { Molecular Weight: } 440.45 \mathrm{~g} / \mathrm{mol} \\
\text { Mechanism: Candesartan is an angiotensin receptor antagonist, blocking vasoconstriction and the } \\
\text { aldosterone-secreting effects (reabsorption of sodium and water) of angiotensin II. } \\
\text { Effect: Angiotensin II Receptor Antagonists. }\end{array}$ & $\begin{array}{l}\text { Mechanistic genes: } A C E, A G T, A G T R 1, B D K R B 2 \text {, } \\
\text { NOS3, PTGS1, TGFB1 } \\
\text { Metabolic genes } \\
\text { Substrate: CYP1A1, CYP2C8, CYP2C9, CYP11B2, } \\
\text { UGT1A3, UGT1A5, UGT2B7 } \\
\text { Transporter genes: } A B C B 1, A B C G 2\end{array}$ \\
\hline
\end{tabular}


Table 2. Cont

\begin{tabular}{|c|c|c|}
\hline \multicolumn{3}{|c|}{ Hypotensive Agents } \\
\hline \multicolumn{3}{|c|}{ Renin-Angiotensin-Aldosterone System Inhibitors } \\
\hline \multicolumn{3}{|c|}{ Angiotensin-Converting Enzyme Inhibitors } \\
\hline Drug & Properties & Pharmacogenetics \\
\hline & $\begin{array}{l}\text { Name: Irbesartan } \\
\text { IUPAC Name: } 1,3 \text {-Diazaspiro[4.4]non-1-en-4-one, } \\
\text { 2-butyl-3-[[2'-(1H-tetrazol-5-yl][1,1'-biphenyl]-4-yl]methyl]- } \\
\text { Molecular Formula: } \mathrm{C}_{25} \mathrm{H}_{28} \mathrm{~N}_{6} \mathrm{O} \\
\text { Molecular Weight: } 428.53 \mathrm{~g} / \mathrm{mol} \\
\text { Mechanism: Irbesartan binds to AT1 angiotensin II receptor. This binding prevents angiotensin II } \\
\text { from binding to receptor, thereby blocking the vasoconstriction and aldosterone-secreting effects of } \\
\text { angiotensin II. } \\
\text { Effect: Angiotensin II Receptor Antagonists. }\end{array}$ & $\begin{array}{l}\text { Mechanistic genes: } A D R A 1 A, A G T, A G T R 1, A P O B \text {, } \\
\text { BDKRB2, JUN, LDLR, NOS3, PTGS1, TGFB1 } \\
\text { Metabolic genes } \\
\text { Substrate: CYP1A2, CYP2C8, CYP2C9, CYP2D6, } \\
\text { CYP3A4, CYP3A5, CYP11B2, UGT1A3 } \\
\text { Transporter genes: ABCB1 ABCG2 } \\
\text { Pleiotropic genes: APOE }\end{array}$ \\
\hline k+ & $\begin{array}{l}\text { Name: Losartan Potassium } \\
\text { IUPAC Name: } 1 H \text {-Imidazole-5-methanol, } \\
\text { 2-butyl-4-chloro-1-[[2'-(1H-tetrazol-5-yl)[1,1'-biphenyl]-4-yl]methyl]-, monopotassium salt } \\
\text { Molecular Formula: } \mathrm{C}_{22} \mathrm{H}_{22} \mathrm{ClKN}_{6} \mathrm{O} \\
\text { Molecular Weight: } 461.00 \mathrm{~g} / \mathrm{mol} \\
\text { Mechanism: As a selective and competitive nonpeptide angiotensin II receptor antagonist, losartan } \\
\text { blocks vasoconstrictor and aldosterone-secreting effects of angiotensin II. Losartan increases urinary } \\
\text { flow rate and, in addition to being natriuretic and kaliuretic, increases excretion of chloride, } \\
\text { magnesium, uric acid, calcium, and phosphate. } \\
\text { Effect: Angiotensin II Receptor Antagonists. }\end{array}$ & $\begin{array}{l}\text { Mechanistic genes: } A C E, A D D 1, A G T, A G T R 1, A G T R 2, \\
A L B, B D K R B 2, E D N 1, F O S, M M P 2, N O S 3, P D G F R B, \\
T G F B 1 \\
\text { Metabolic genes } \\
\text { Substrate: CYP1A2, CYP2C8, CYP2C9, CYP2C19, } \\
\text { CYP3A4, CYP3A5, CYP11B2, UGT1A1, UGT1A3, } \\
\text { UGT1A10, UGT2B7, UGT2B17 } \\
\text { Transporter genes: } A B C B 1, A B C G 2, S L C 2 A 9, \\
\text { SLC22A6, SLC22A12 } \\
\text { Pleiotropic genes: } T N F\end{array}$ \\
\hline
\end{tabular}


Table 2. Cont

\begin{tabular}{|c|c|c|}
\hline \multicolumn{3}{|c|}{ Hypotensive Agents } \\
\hline \multicolumn{3}{|c|}{ Renin-Angiotensin-Aldosterone System Inhibitors } \\
\hline \multicolumn{3}{|c|}{ Angiotensin-Converting Enzyme Inhibitors } \\
\hline Drug & Properties & Pharmacogenetics \\
\hline & $\begin{array}{l}\text { Name: Olmesartan Medoxomil } \\
\text { IUPAC Name: } 1 H \text {-Imidazole-5-carboxylic acid, } \\
\text { 4-(1-hydroxy-1-methylethyl)-2-propyl-1-[[2'-(1H-tetrazol-5-yl) [1,1'-biphenyl]-4-yl]methyl]-, } \\
\text { (5-methyl-2-oxo-1,3-dioxol-4-yl)methyl ester } \\
\text { Molecular Formula: } \mathrm{C}_{29} \mathrm{H}_{30} \mathrm{~N}_{6} \mathrm{O}_{6} \\
\text { Molecular Weight: } 558.59 \mathrm{~g} / \mathrm{mol}^{\prime} \\
\text { Mechanism: Blocks vasoconstrictor and aldosterone-secreting effects of angiotensin II. Interacts } \\
\text { reversibly at AT1 and AT2 receptors and has slow dissociation kinetics (has greater affinity for AT1 } \\
\text { receptor). Olmesartan increases urinary flow rate and, besides being natriuretic and kaliuretic, } \\
\text { increases excretion of chloride, magnesium, uric acid, calcium, and phosphate. } \\
\text { Effect: Angiotensin II Receptor Antagonists. }\end{array}$ & $\begin{array}{l}\text { Mechanistic genes: } A G T R 1, A C E 2, E D N 1, T G F B 1 \\
\text { Metabolic genes } \\
\text { Substrate: } C M B L, C Y P 2 C 9 \\
\text { Transporter genes: } A B C B 1, A B C C 2, A B C G 2, S L C O 1 B 1 \text {, } \\
\text { SLCO1B3, SLCO1A2, SLC22A8 } \\
\text { Pleiotropic genes: } A P O E\end{array}$ \\
\hline & $\begin{array}{l}\text { Name: Telmisartan } \\
\text { IUPAC Name: }\left[1,1^{\prime}-\text {-Biphenyl]-2-carboxylic acid, }\right. \\
4^{\prime}-\left[\left(1,4^{\prime} \text {-dimethyl-2'-propyl[2,6'-bi-1H-benzimidazol]-1'-yl)methyl]- }\right.\right. \\
\text { Molecular Formula: } \mathrm{C}_{33} \mathrm{H}_{30} \mathrm{~N}_{4} \mathrm{O}_{2} \\
\text { Molecular Weight: } 514.62 \mathrm{~g} / \mathrm{mol}^{\prime} \\
\text { Mechanism: A nonpeptide } \mathrm{AT}_{1} \text { angiotensin II receptor antagonist. This binding prevents angiotensin } \\
\text { II from binding to receptor thereby blocking vasoconstriction and aldosterone-secreting effects of } \\
\text { angiotensin II. } \\
\text { Effect: Angiotensin II Receptor Antagonists. }\end{array}$ & $\begin{array}{l}\text { Mechanistic genes: } A C E, A G T, A G T R 1, B D K R B 2 \text {, } \\
E R A P 1, P P A R G \\
\text { Metabolic genes } \\
\text { Substrate: } C Y P 2 C 9, C Y P 2 C 19, C Y P 11 B 2, U G T 1 A 1 \\
\text { Transporter genes: } A B C B 1, A B C C 2, A B C G 2\end{array}$ \\
\hline & $\begin{array}{l}\text { Name: Valsartan } \\
\text { IUPAC Name: L-Valine, } \mathrm{N}-(1 \text {-oxopentyl)- } \mathrm{N} \text {-[[2'-(1H-tetrazol-5-yl)[1,1'-biphenyl]-4-yl]methyl]- } \\
\text { Molecular Formula: } \mathrm{C}_{24} \mathrm{H}_{29} \mathrm{~N}_{5} \mathrm{O}_{3} \\
\text { Molecular Weight: } 435.52 \mathrm{~g} / \mathrm{mol}^{\prime} \\
\text { Mechanism: Displaces angiotensin II from } \mathrm{AT}_{1} \text { receptor and produces its blood pressure-lowering } \\
\text { effects by antagonizing } \mathrm{AT}_{1} \text {-induced vasoconstriction, aldosterone release, catecholamine release, } \\
\text { arginine vasopressin release, water intake, and hypertrophic responses. } \\
\text { Effect: Angiotensin II Receptor Antagonists. }\end{array}$ & $\begin{array}{l}\text { Mechanistic genes: } A C E, A G T, A G T R 1, A L B, B D K R B 2 \text {, } \\
\text { ERAP1, GNB3, } \\
\text { Metabolic genes } \\
\text { Substrate: CYP2C9, CYP2C19, CYP2D6, CYP3A4, } \\
\text { CYP3A5, CYP11B2 } \\
\text { Transporter genes: } A B C C 2, \text { SLCO1B1, SLCO1B3 }\end{array}$ \\
\hline \multicolumn{3}{|c|}{ 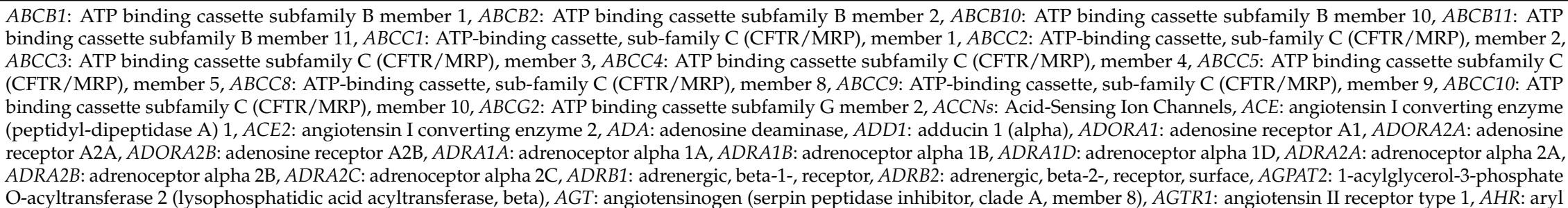 } \\
\hline
\end{tabular}


hydrocarbon receptor, $A K R 1 C 4$ : aldo-keto reductase family 1, member C4 (chlordecone reductase; 3-alpha hydroxysteroid dehydrogenase, type I; dihydrodiol dehydrogenase 4), $A L B$ : albumin, $A L D H 2$ : aldehyde dehydrogenase 2 family (mitochondrial), $A L O X 5$ : arachidonate 5-lipoxygenase, $A O C 3$ : amine oxidase, copper containing 3, APC: adenomatous polyposis coli, APOB: apolipoprotein B, APOE: apolipoprotein E, APP: amyloid beta (A4) precursor protein, AR: androgen receptor, ARG1: arginase, liver, ASIC1: acid sensing ion channel subunit 1, ASIC2: acid sensing ion channel subunit 2, ATP1A1: ATPase $\mathrm{Na}^{+} / \mathrm{K}^{+}$transporting subunit alpha 1, AVPR1A: arginine vasopressin receptor 1A, AVPR2: arginine vasopressin receptor 2, BDKRB2: bradykinin receptor B2, BIRC5 (NAIP): NLR family apoptosis inhibitory protein, CA1: carbonic anhydrase 1, CA2: carbonic anhydrase 2, CA4: carbonic anhydrase 4, CA9: carbonic anhydrase 9, CA12: carbonic anhydrase 12, CACNA1A: calcium channel, voltage-dependent, alpha-1 subunit, $C A C N A 1 B$ : calcium channel, voltage-dependent, beta-1 subunit, $C A C N A 1 C$ : calcium channel, voltage-dependent, alpha-1 subunit, $C A C N A 1 D$ : calcium channel, voltage-dependent, delta-1 subunit, $C A C N A 1 F$ : calcium channel, voltage-dependent subunit alpha1 $\mathrm{F}_{,}$ CACNA1G: calcium channel, voltage-dependent subunit alpha1 G, CACNA1I: calcium channel, voltage-dependent subunit alpha1 I, CACNA1S: calcium channel, voltage-dependen subunit alpha1 S, CACNA2D1: calcium voltage-gated channel auxiliary subunit alpha 2 delta 1, CACNB1: calcium voltage-gated channel auxiliary subunit beta 1, CACNB2: calcium voltage-gated channel auxiliary subunit beta 2, CACNB3: calcium voltage-gated channel auxiliary subunit beta 3, CACNB4: calcium voltage-gated channel auxiliary subunit beta 4 CACNG1: calcium voltage-gated channel auxiliary subunit gamma 1, CALM1: calmodulin 1, CCL23: C-C motif chemokine ligand 23, CEL: carboxyl ester lipase, CES1: carboxylesterase 1, CFH: complement factor H, CFTR: cystic fibrosis transmembrane conductance regulator (ATP-binding cassette sub-family C, member 7), CHAT: choline acetyltransferase, CHRM1: cholinergic receptor muscarinic 1, CHRM2: cholinergic receptor muscarinic 2, CHRM3: cholinergic receptor muscarinic 3, CHRM4: cholinergic receptor muscarinic 4, CHRM5: cholinergic receptor muscarinic 5, CHRNA1: cholinergic receptor, nicotinic, alpha 1, CHRNA2: cholinergic receptor, nicotinic, alpha 2, CHRNA4: cholinergic receptor, nicotinic, alpha 4, CHRNA7: cholinergic receptor, nicotinic, alpha 7, CHRNB2: cholinergic receptor, nicotinic, beta 2, CMBL: carboxymethylenebutenolidase homolog, COL1A1: collagen, type I, alpha 1, COMT: catechol-O-methyltransferase, CPNE1: copine 1, CTNNB1: catenin beta 1, CYP1A1: cytochrome P450, family 1, subfamily A, polypeptide 1, CYP1A2: cytochrome P450 family 1 subfamily A member 2, CYP1B1: cytochrome P450 family 1 subfamily B member 1, CYP2C8: cytochrome P450, family 2, subfamily C, polypeptide 8, CYP2C9: cytochrome P450, family 2, subfamily C, polypeptide 9, CYP2D6: cytochrome P450, family 2, subfamily D, polypeptide 6, CYP2E1: cytochrome P450, family 2, subfamily E, polypeptide 1, CYP2J2: cytochrome P450, family 2, subfamily J, polypeptide 2, CYP3A4: cytochrome P450 family 3 subfamily A member 4, CYP3A5: cytochrome P450 family 3 subfamily A member 5, CYP3A7: cytochrome P450 family 3 subfamily A member 7, CYP7A1: cytochrome P450, family 7, subfamily A, polypeptide 1, CYP11B1: cytochrome P450, family 11, subfamily B, polypeptide 1, CYP11B2: cytochrome P450, family 11, subfamily B, polypeptide 2, CYP17A1: cytochrome P450, family 17, subfamily A, polypeptide 1, CYP19A1: cytochrome P450, family 19, subfamily A, polypeptide 1, CYPs: cytochrome P450 family, DDC: dopa decarboxylase, DRD1: dopamine receptor D1, DRD2: dopamine receptor D2, DRD5: dopamine receptor D5, EDN1: endothelin 1, EDNRA: endothelin receptor type A, EDNRB: endothelin receptor type B, ERAP1: endoplasmic reticulum aminopeptidase 1, ERBB2: v-erb-b2 erythroblastic leukemia viral oncogene homolog 2 , neuro/glioblastoma derived oncogene homolog (avian), ESR1: estrogen receptor 1, FOS: FBJ murine osteosarcoma viral oncogene homolog, GLUL: glutamine synthetase, GNB3: guanine nucleotide binding protein (G protein), beta polypeptide 3, GSTA1: glutathione S-transferase alpha 1, GSTA2: glutathione S-transferase alpha 2, GSTM1: glutathione S-transferase mu 1, GSTP1: glutathione S-transferase pi 1, GSTs: glutathione S-transferases, GSTT1: glutathione $S$-transferase theta 1, GUCY1A2: guanylate cyclase 1 soluble subunit alpha 2, HBB: hemoglobin, beta, HDAC2: histone deacetylase 2, HFE: hemochromatosis, HIF1A: hypoxia inducible factor 1 alpha subunit, $H L A-A$ : major histocompatibility complex, class I, A, HLA-B: major histocompatibility complex, class I, B, HM13: histocompatibility minor 13,HNF4: hepatocyte nuclear factor 4, alpha, HRH1: histamine receptor H1, HRH2: histamine receptor H2, IDO1: indoleamine 2,3-dioxygenase 1, IL10: interleukin 10, IL1B: interleukin 1, beta, IL6: interleukin 6 (interferon, beta 2), JUN: Jun proto-oncogene, AP-1 transcription factor subunit, KCNE1: potassium voltage-gated channel subfamily E member 1, KCNH2: potassium voltage-gated channel subfamily H member 2, KCNJ1: potassium voltage-gated channel subfamily J member 1, KCNJ11: potassium inwardly-rectifying channel, subfamily J, member 11, KCNJ5: potassium voltage-gated channel subfamily J member 5, KCNMA1: potassium calcium-activated channel subfamily $\mathrm{M}$ alpha 1, KCNQ1: potassium inwardly-rectifying channel, subfamily Q, member 1, KDR: kinase insert domain receptor (a type III receptor tyrosine kinase), $L E F 1$ : lymphoid enhancer binding factor 1, $L D L R$ : low density lipoprotein receptor, $L T A 4 H$ : leukotriene A4 hydrolase, $M A O A$ : monoamine oxidase $\mathrm{A}, M A O B$ : monoamine oxidase B, MGMT: O-6-methylguanine-DNA methyltransferase, MMP2: matrix metallopeptidase 2, MMP3: matrix metallopeptidase 3, MMP9: matrix metallopeptidase 9, MTHFR: methylenetetrahydrofolate reductase, MT1A: metallothionein 1A, mtlD: mannitol-1-phosphate dehydrogenase, NAD-dependent, MTR: 5-methyltetrahydrofolate-homocysteine methyltransferase, NAT2: $N$-acetyltransferase 2 (arylamine $N$-acetyltransferase), NOMO1: NOMO nodal modulator 1, NOS3: nitric oxide synthase 3 (endothelial cell), NPPA: natriuretic peptide precursor A, NPR1: natriuretic receptor precursor 1, NR112: nuclear receptor subfamily 1, group I, member 2, NR3C1: nuclear receptor subfamily 3, group C, member 1 (glucocorticoid receptor), NR3C2: nuclear receptor subfamily 3, group C, member 2 (glucocorticoid receptor), P2RY12: purinergic receptor P2Y, G-protein coupled, 12, ORM1: orosomucoid 1, P4HA1: prolyl 4-hydroxylase subunit alpha 1, PARP1: poly(ADP-ribose) polymerase 1, PDE1C: phosphodiesterase 1C, PDE1A: phosphodiesterase 1A, PDE3A: phosphodiesterase 3A PDE1B: phosphodiesterase 1B, PDE4A: phosphodiesterase 4A, PDE4B: phosphodiesterase 4B, PDE5A: phosphodiesterase 5A, cGMP-specific, $P D E 6 A$ : phosphodiesterase 6A, cGMP-specific 
PDE6G: phosphodiesterase 6G, PDE6H: phosphodiesterase 6H, PDE10A: phosphodiesterase 10A, PDE11A: phosphodiesterase 11A, PDGFRB: platelet-derived growth factor receptor, beta polypeptide, $P G D$ : phosphogluconate dehydrogenase, $P G R$ : progesterone receptor, $P K D 1$ : polycystin 1, transient receptor potential channel interacting, $P K D 2$ : polycystin 2, transien receptor potential channel interacting, PLAU: plasminogen activator, urokinase, $P L A T$ : plasminogen activator, tissue type, PPARD: peroxisome proliferator-activated receptor-delta, PPARG: peroxisome proliferator-activated receptor-gamma, PTGERs: prostaglandin E receptors, PTGIR: prostaglandin I2 (prostacyclin) receptor (IP), PTGS1: prostaglandin-endoperoxide synthase 1 (prostaglandin G/H synthase and cyclooxygenase), PTGS2: prostaglandin-endoperoxide synthase 2 (prostaglandin G/H synthase and cyclooxygenase), RCAN1: regulator of calcineurin 1, REN: renin, RIC3: RIC3 acetylcholine receptor chaperone, SCN5A: sodium voltage-gated channel alpha subunit 5, SCNN1A: sodium channel epithelial 1 alpha subunit, SCNN1B: sodium channel epithelial 1 beta subunit, SCNN1D: sodium channel epithelial 1 delta subunit, SCNN1G: sodium channel, nonvoltage-gated 1, gamma, SCNs: sodium channels, nonvoltage-gated, SFRP4: secreted frizzled related protein 4, SHBG: sex hormone binding globulin, SLC6A2: solute carrier family 6 (neurotransmitter transporter, noradrenalin), member 2, SLC6A4: solute carrier family 6 member 4, SLC9A1: solute carrier family 9 member 1, SLC10A1: solute carrier family 10 member 1, SLC12A1: solute carrier family 12 member 1, SLC12A2: solute carrier family 12 member 2, SLC12A3: solute carrier family 12, member 3, SLC12A4: solute carrier family 12, member 4, SLC14A1: solute carrier family 14 member 1, SLC14A2: solute carrie family 14 member 2, SLC15A1: solute carrier family 15 member 1, SLC15A2: solute carrier family 15 member 2, SLC18A2: solute carrier family 18, member 2, SLC19A1: solute carrier famil 19 (folate transporter), member 1, SLC22A1: solute carrier family 22 member 1, SLC22A2: solute carrier family 22 member 2, SLC22A3: solute carrier family 22 member 3, SLC22A4: solute carrier family 22 member 4, SLC22A5: solute carrier family 22 member 5, SLC22A6: solute carrier family 22 member 6, SLC22A7: solute carrier family 22 member 7, SLC22A8: solute carrier family 22 member 8, SLC22A11: solute carrier family 22 member 11, SLC22A16: solute carrier family 22 member 16, SLC29A: solute carrier family 29, SLCO1A2: solute carrier organic anion transporter family, member 1A2, SLCO1B1: solute carrier organic anion transporter family, member 1B1, SLCO1B3: solute carrier organic anion transporter family, member 1B3, SRD5A1: steroid 5 alpha-reductase 1, SULT1A1: sulfotransferase family, cytosolic, 1A, phenol-preferring, member 1, SULT1A3: sulfotransferase family, cytosolic, 1A, phenol-preferring, member 3, TGFB1: transforming growth factor, beta 1, TNF: tumor necrosis factor (TNF superfamily, member 2, TP53: tumor protein p53, TTLL3: tubulin tyrosine ligase like 3, UGT1A1: UDP glucuronosyltransferase 1 family, polypeptide A1, UGT1A3: UDP glucuronosyltransferase 1 family, polypeptide A3, UGT1A6: UDP glucuronosyltransferase 1 family, polypeptide A6, UGT1A9: UDP glucuronosyltransferase 1 family, polypeptide A9, UGT2B7: UDP glucuronosyltransferase 2 family, polypeptide B7, UGT2B17: UDP glucuronosyltransferase 2 family, polypeptide B17, UGTs: UDP glucuronosyltransferase family, USP5: ubiquitin specific peptidase 5 (isopeptidase T), WNK1: WNK lysine deficient protein kinase 1. 
APOE- Related Blood Pressure Response to Hypotensive Treatment

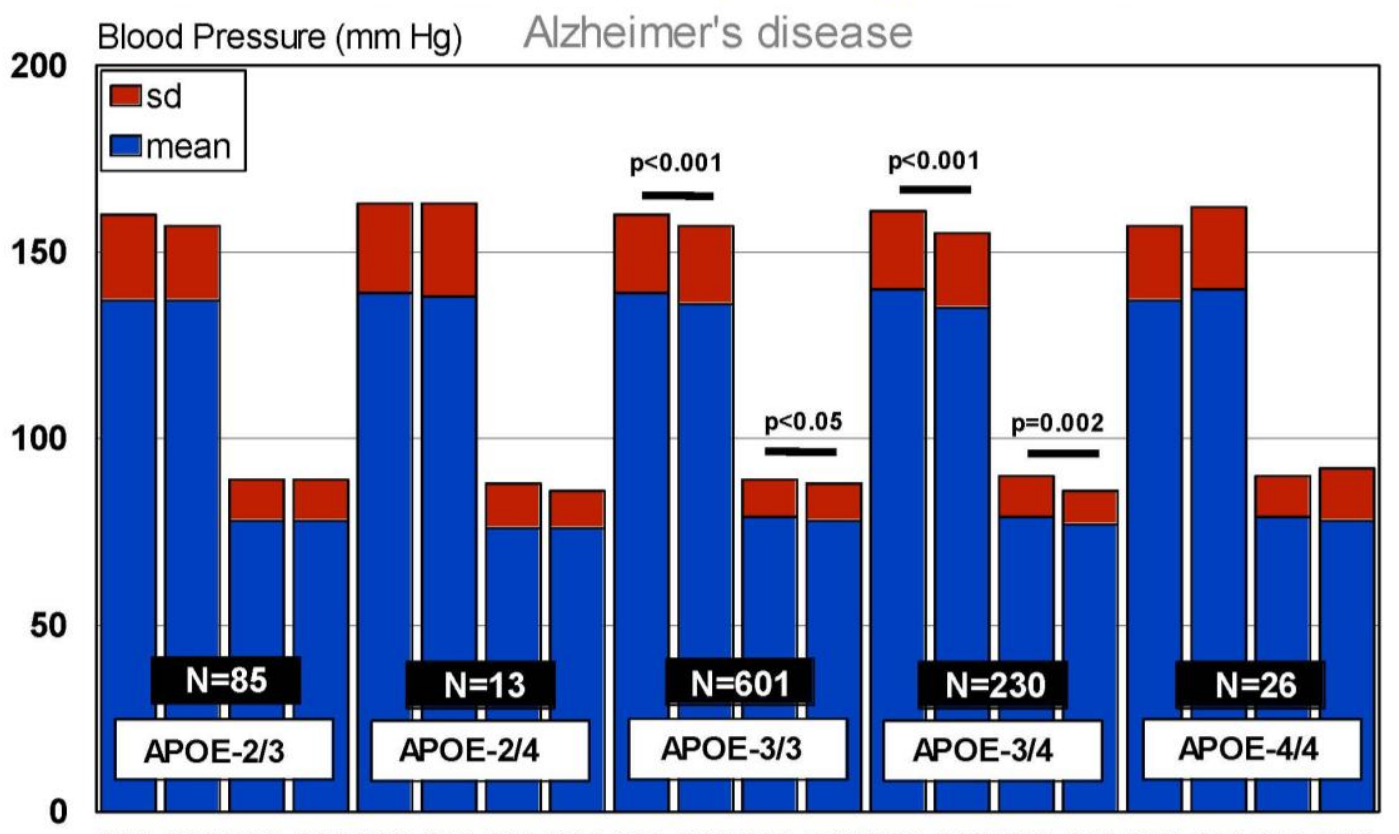

SBPb SBPt DBPb DBPt SBPb SBPt DBPb DBPt SBPb SBPt DBPb DBPt SBPb SBPt DBPb DBPt SBPb SBPt DBPb DBPt

Figure 14. APOE-related blood pressure response to a hypotensive treatment. SBPb: Systolic blood pressure. SBPt: Systolic blood pressure (Enalapril, 10-20 mg/day). DBP: Diastolic blood pressure.

NOS3-Related Blood Pressure Response to Hypotensive Treatment

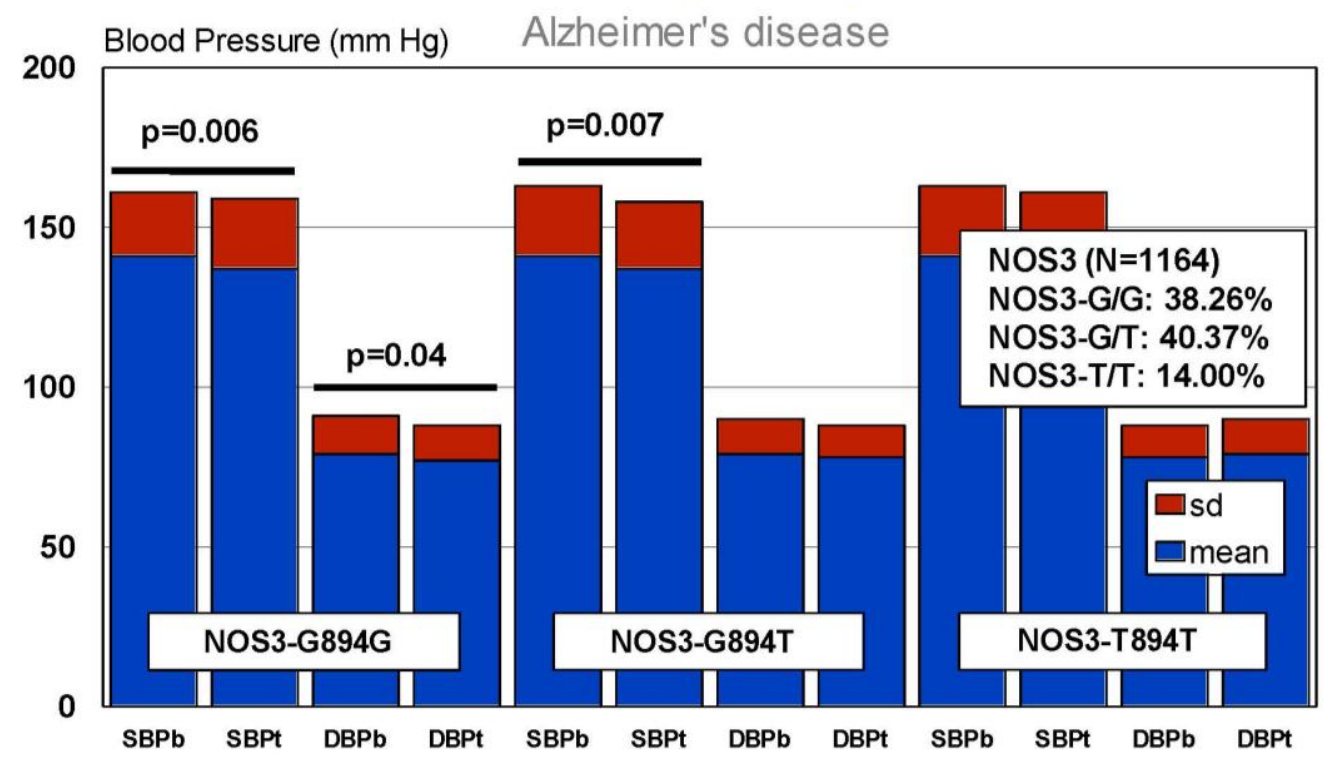

Figure 15. NOS3-related blood pressure response to a hypotensive treatment. SBPb: Systolic blood pressure. SBPt: Systolic blood pressure (Enalapril, 10-20 mg/day). DBP: Diastolic blood pressure. 


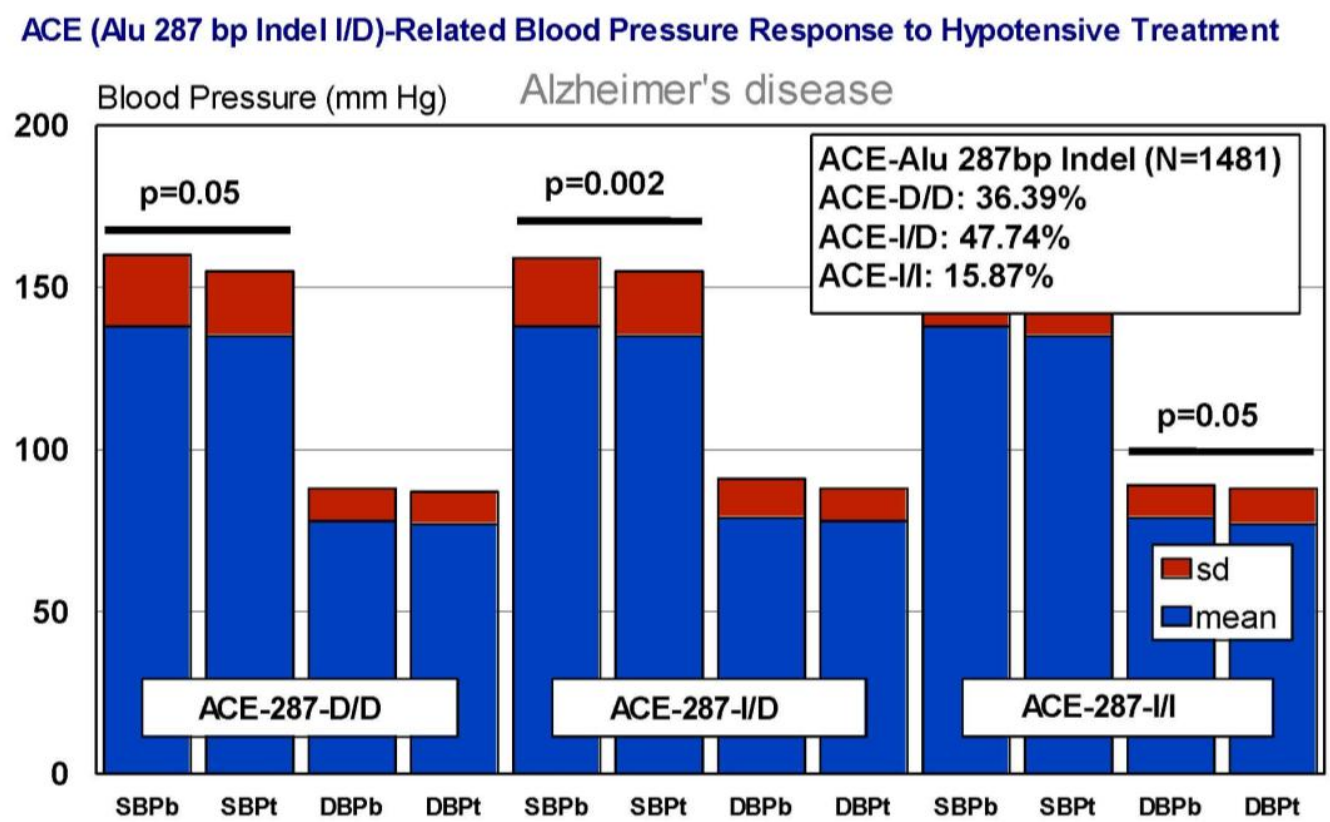

Figure 16. $A C E$-related blood pressure response to a hypotensive treatment. SBPb: Systolic blood pressure. SBPt: Systolic blood pressure (Enalapril, 10-20 mg/day). DBP: Diastolic blood pressure.

\section{AGT-G9360ARelated Blood Pressure Response to Hypotensive Treatment}

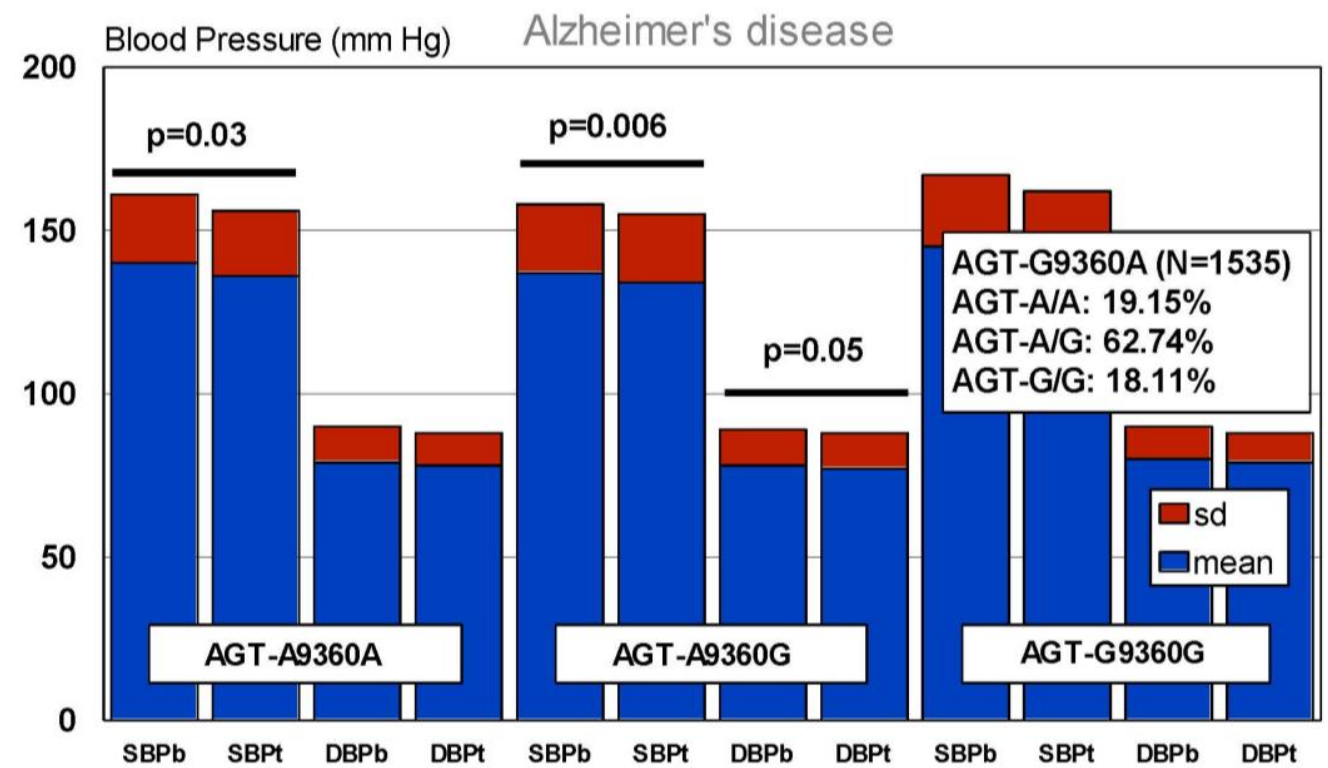

Figure 17. AGT-related blood pressure response to a hypotensive treatment. SBPb: Systolic blood pressure. SBPt: Systolic blood pressure (Enalapril, 10-20 mg/day). DBP: Diastolic blood pressure.

Enalapril [L-Proline,1-[N-[1-(ethoxycarbonyl)-3-phenylpropyl]-L-alanyl]-,(S)-,(Z)-2-butenedioate $(1: 1)]$ is a competitive ACE inhibitor that prevents the conversion of angiotensin I to angiotensin II, a potent vasoconstrictor, resulting in lower levels of angiotensin II, which causes an increase in plasma renin activity and a reduction in aldosterone secretion [36]. ACE, ADRB2, AGT, AGTR1, BDKRB2, and NOS3 are mechanistic genes that regulate the effects of Enalapril. Forty-eight genes show evidence of involvement in blood pressure regulation and 6 new signals of association in or near HSPB7, TNXB, LRP12, LOC283335, SEPT9, and AKT2 have been reported, with new replication evidence for EBF2 and NFKBIA [69]. This ACE inhibitor is an apparent substrate of CYP3A4/5 enzymes and is transported 
by SLC15A1, SLC22A6, SLC22A7, SLC22A8, and SLCO1A2 gene products [36]. Interestingly, we did not find any effect of CYP3A4/5 variants in EM, IM and RMs on blood pressure changes after one month of treatment with Enalapril in AD patients. Therefore, further studies seem to be necessary to elucidate the role of CYP variants in Enalapril metabolism.

Basic and clinical studies suggest that some hypotensive agents may be beneficial in AD [70,71]. Angiotensin-converting enzyme inhibitors (ACEIs) and angiotensin receptor blockers (ARBs) are common anti-hypertensive treatments, but have differential effects on cortical amyloid [70].

The renin angiotensin system (RAS) is directly associated with hypertension, and overactivity of RAS may perpetuate AD-related brain inflammation. It has been reported that Candesartan, an angiotensin type 1 receptor (AT1R) blocker (ARB), may prevent astrocyte and microglial activation and neuroinflammation in the brain of hypertensive rats. This hypotensive agent, by activating Wnt/ $\beta$-catenin signaling, promotes neurogenesis during the hypertensive state, prevents astrocyte and microglial activation, and improves hippocampal neurogenesis in hypertensive state, independently of its classical hypotensive effect [71]. In humans with mild cognitive impairment (MCI) or AD, the individuals treated with ARBs showed greater hippocampal volumes and brain parenchymal fraction than those treated with ACEIs. ARBs were also associated with significantly better performance in tests of episodic and verbal memory, language, and executive function [70].

\section{EKG Conditions and Blood Pressure in Alzheimer's Disease}

Cardiovascular drugs, associated or not with anti-hypertensive agents, are frequently given to elderly subjects and demented patients. Approximately $50 \%$ of AD patients show some kind of heart disorder susceptible to pharmacological treatment. In our AD population, we studied the frequency of cardiovascular disorders in comparison with the control population. We divided both cohorts according to a normal, borderline, or abnormal electrocardiogram (EKG). Among AD patients, $48.56 \%$ showed a normal EKG, $8.00 \%$ a borderline EKG, and $43.44 \%$ an abnormal EKG. In the control population, the EKG was normal in $60 \%$ of the cases, borderline in $11.47 \%$, and abnormal in $25.33 \%$ (Odds ratio (OR) 1.92; 95\% Confidence interval (CI) 1.63-2.26; $p<0.0005$ AD vs. controls) (Figure 18). Interestingly enough, SBP levels, but not DBP levels, were significantly higher in AD than in controls, unrelated to normal, borderline, or abnormal EKGs (Figure 19), probably suggesting that hypertension and heart disease are independent risk factors for $\mathrm{AD}$ and vascular dementia [45]. In fact, recent studies indicate that myocardial infarction is associated with a higher risk of dementia [72]; prevalent and incident cardiovascular disorders predict cognitive decline [73]; and a consistent inverse association was observed between systolic blood pressure and AD [74]. However, in our casuistic, SBP tends to be higher in $\mathrm{AD}$ as compared to controls with no history of dementia. Furthermore, small-vessel disease-related white matter lesions are associated with gray matter atrophy and with cognitive performance [75]; and hypertension is a contributing factor to vascular microlesions in AD brains. 
EKG in patients with Alzheimer's disease and controls

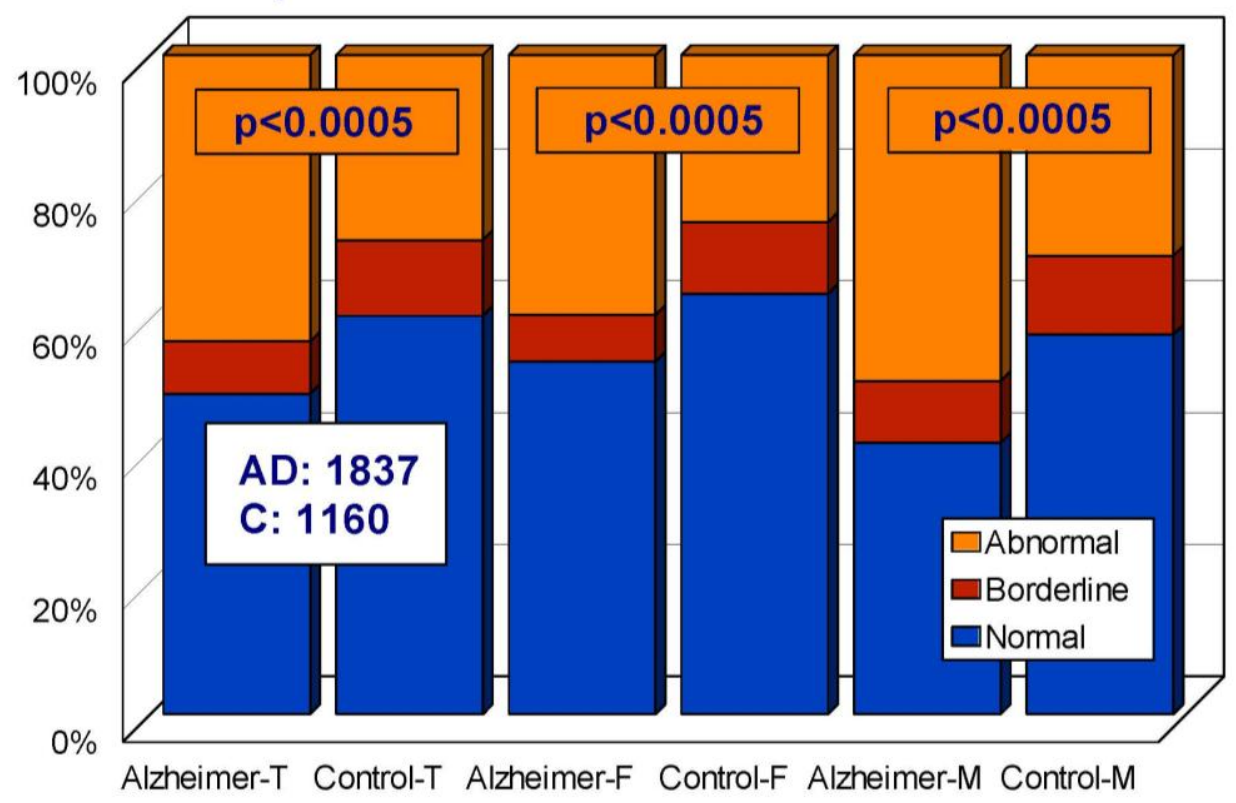

Figure 18. Electrocardiogram (EKG) in patients with Alzheimer's disease and controls. T: Total; F: Females; M: Males.

\section{EKG-Related Systolic Blood Pressure}

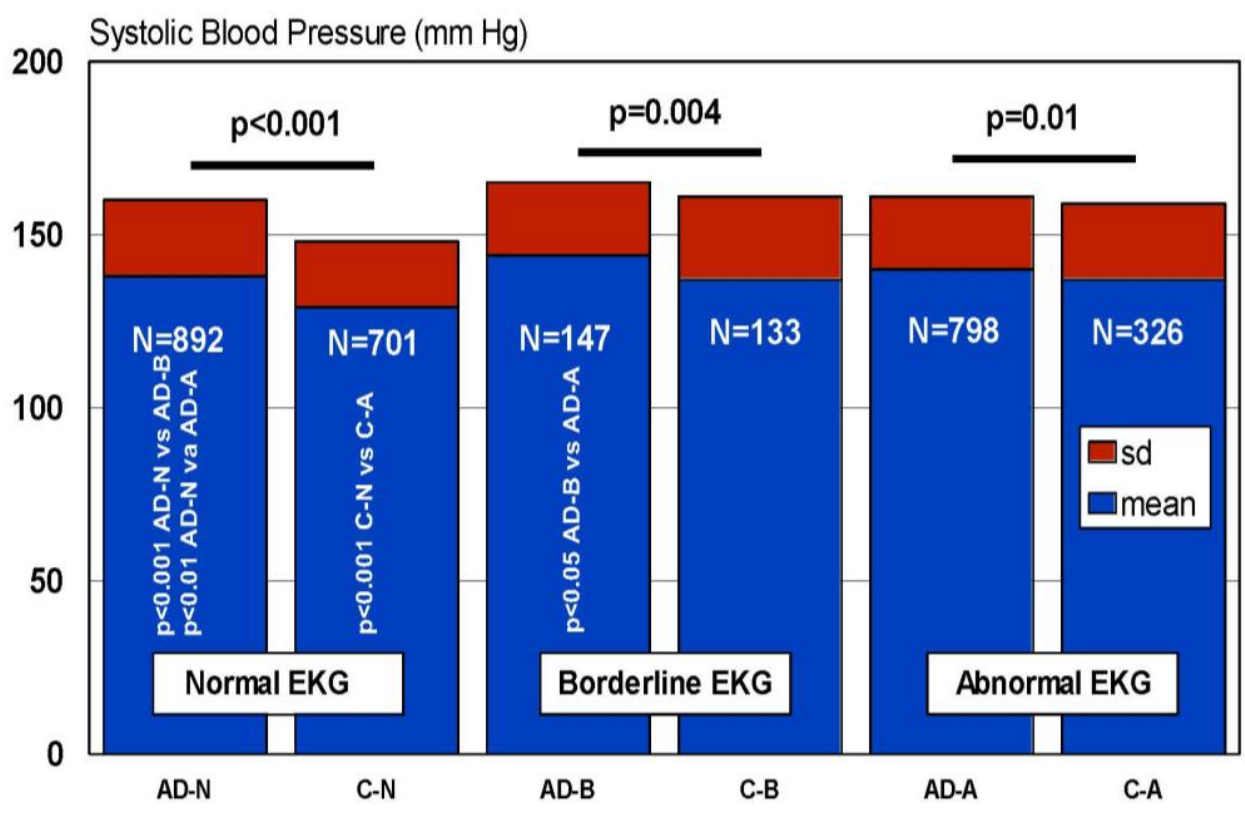

Figure 19. Electrocardiogram (EKG)-related systolic blood pressure in patients with Alzheimer's disease and controls. AD: Alzheimer's disease. C: Control. N: Normal. B: Borderline. A: Abnormal. AD-N vs. AD-B, $p<0.001$; AD-N vs. AD-A, $p<0.01$; C-N vs. C-A, $p<0.001$; AD-B vs. AD-A, $p>0.05$.

\section{Concluding Remarks}

According to the present examples and abundant data collected from the international literature [36], it seems clear that cardio-cerebrovascular risk factors, such as blood pressure changes, hypercholesterolemia, and heart disorders may contribute to exacerbating the disease process in $\mathrm{AD}$ patients. Most of these medical conditions require pharmacological intervention with drugs that 
can interact among themselves and with anti-dementia drugs as well. Additionally, the therapeutic response to conventional drugs is genotype-dependent, but routine pharmacogenetic studies are scarcely performed; and over $90 \%$ of AD patients simultaneously receive different types of drugs for concomitant disorders with a high risk of drug-drug interactions and ADRs. From a practical perspective, in order to help physicians in their daily clinical practice, the implementation of relatively simple protocols of pharmacogenetics would be of great utility [17]. Examples derived from the present results suggest (i) that the dose of anti-dementia drugs in APOE-4 carriers and in CYP2D6-PMs and CYP2D6-UMs be adjusted, (ii) that the dose of statins in CYP3A4-IMs be reduced and statins metabolized via CYP enzymes in CYP3A4-RMs avoided due to inefficacy, and (iii) that the dose of ACE inhibitors (or select a more effective anti-hypertensive agent) in APOE-4/4, NOS3-T/T, and GT-G/G carriers be adjusted.

Some reflections might be necessary for improving the multifactorial therapeutic intervention in a complex disorder such as AD: (i) a better characterization of the roles played in drug efficacy and safety by genes involved in the pharmacogenomic network is highly desirable; (ii) since most genes are under the influence of the epigenetic machinery, pharmacoepigenomics is becoming an attractive field that deserves special attention, and some epigenetic drugs might also be helpful in selected AD cases, although at present most epigenetic drugs pose technical problems (bioavailability, toxicity, and brain penetration) [26,34,76]; (iii) drug-drug interactions represent a problematic issue in over $80 \%$ of AD patients; to palliate this difficulty, simple pharmacogenetic protocols should be introduced in the clinical setting to help physicians in their daily prescription activity, to minimize ADRs; (iv) since the neurodegenerative process underlying $\mathrm{AD}$ neuropathology starts 20-30 years before the onset of the disease, novel therapeutics should be addressed to prevent premature neuronal death (symptomatic drugs have proven to be poorly effective), and a better knowledge of drugs with potential neurodegenerative effects after chronic treatments is also necessary to limit their inappropriate use; (v) specific biomarkers for AD are necessary in three different contexts: predictive markers before disease onset, early diagnosis in initial stages, and drug monitoring (in both preventive and/or therapeutic strategies); and (vi) educational programs are fundamental for physicians to be aware of the usefulness of pharmacogenomics to prescribe more accurately, to avoid adverse reactions and to optimize the limited therapeutic resources available for the treatment of dementia $[8,16,17]$.

Acknowledgments: Studies reported in this review were supported by the Research Division of EuroEspes Biomedical Research Center, Corunna, Spain.

Conflicts of Interest: R.C. is President of EuroEspes, S.A., and EuroEspes Biotechnology (Ebiotec), and A.M., J.C.C., P.C. and O.T. are staff members of the Medical Research Division of EuroEspes Biomedical Research Center.

\section{References}

1. Cacabelos, R. The application of functional genomics to Alzheimer's disease. Pharmacogenomics 2003, 4, 597-621. [CrossRef] [PubMed]

2. Cacabelos, R. Molecular pathology and pharmacogenomics in Alzheimer's disease: Polygenic-related effects of multifactorial treatments on cognition, anxiety, and depression. Methods Find. Exp. Clin. Pharmacol. 2007, 29 (Suppl. A), 1-91. [PubMed]

3. Cacabelos, R. Pharmacogenomics and therapeutic strategies for dementia. Expert Rev. Mol. Diagn. 2009, 9, 567-611. [CrossRef] [PubMed]

4. Cacabelos, R.; Fernández-Novoa, L.; Corzo, L.; Amado, L.; Pichel, V.; Lombardi, V.; Kubota, Y. Phenotypic profiles and functional genomics in Alzheimer's disease and in dementia with a vascular component. Neurol. Res. 2004, 26, 459-480. [CrossRef] [PubMed]

5. De la Torre, J.C. Cerebral Perfusion Enhancing Interventions: A New Strategy for the Prevention of Alzheimer Dementia. Brain Pathol. 2016, 26, 618-631. [CrossRef] [PubMed]

6. Cacabelos, R.; Goldgaber, D.; Roses, A.D.; Vostrov, A.; Matsuki, H.; Torrellas, C.; Cacabelos, P.; Corzo, D.; Carril, J.C.; Fernández-Novoa, L.; et al. Gene interactions in the Pharmacogenomics of Alzheimer's Disease. Sciforschen Genet. Gene Ther. 2015, 1. [CrossRef] 
7. Cacabelos, R.; Goldgaber, D.; Vostrov, A.; Matsuki, H.; Torrellas, C.; Corzo, D.; Carril, J.C.; Roses, A.D. APOE-TOMM40 in the Pharmacogenomics of dementia. J. Pharmacogenom. Pharmacoproteom. 2014, 5, 135. [CrossRef]

8. Cacabelos, R.; Torrellas, C.; Carrera, I. Opportunities in Pharmacogenomics for the treatment of Alzheimer's Disease. Future Neurol. 2015, 10, 229-252. [CrossRef]

9. Taipale, H.; Koponen, M.; Tanskanen, A.; Tolppanen, A.M.; Tiihonen, J.; Hartikainen, S. Drug use in persons with and without Alzheimer's disease aged 90 years or more. Age Ageing 2016, 45, 900-904. [CrossRef] [PubMed]

10. Cacabelos, R.; Cacabelos, P.; Torrellas, C.; Tellado, I.; Carril, J.C. Pharmacogenomics of Alzheimer's disease: Novel therapeutic strategies for drug development. Methods Mol. Biol. 2014, 1175, 323-556. [PubMed]

11. Cacabelos, R.; Torrellas, C.; Carrera, I.; Cacabelos, P.; Corzo, L.; Fernández-Novoa, L.; Tellado, I.; Carril, J.C.; Aliev, G. Novel Therapeutic Strategies for Dementia. CNS Neurol. Disord. Drug Targets 2016, 15, 141-241. [CrossRef] [PubMed]

12. Wang, J.; Zhao, Z.; Lin, E.; Zhao, W.; Qian, X.; Freire, D.; Bilski, A.E.; Cheng, A.; Vempati, P.; Ho, L.; et al. Unintended effects of cardiovascular drugs on the pathogenesis of Alzheimer's disease. PLoS ONE 2013, 8, e65232. [CrossRef] [PubMed]

13. Montastruc, F.; Gardette, V.; Cantet, C.; Piau, A.; Lapeyre-Mestre, M.; Vellas, B. Potentially inappropriate medication use among patients with Alzheimer disease in the REAL.FR cohort: Be aware of atropinic and benzodiazepine drugs! Eur. J. Clin. Pharmacol. 2013, 69, 1589-1597. [CrossRef] [PubMed]

14. Yuan, J.; Wen, G.; Li, Y.; Liu, C. The occurrence of cerebrovascular atherosclerosis in Alzheimer's disease patients. Clin. Interv. Aging 2013, 8, 581-584. [PubMed]

15. Chen, Y.; Sillaire, A.R.; Dallongeville, J.; Skrobala, E.; Wallon, D.; Dubois, B.; Hannequin, D.; Pasquierthe, F. Lille YOD Study Group. Low Prevalence and Clinical Effect of Vascular Risk Factors in Early-Onset Alzheimer's Disease. J. Alzheimers Dis. 2017, 60, 1045-1054. [CrossRef] [PubMed]

16. Cacabelos, R.; Torrellas, C.; Teijido, O.; Carril, J.C. Pharmacogenetic considerations in the treatment of Alzheimer's Disease. Pharmacogenomics 2016, 17, 1041-1074. [CrossRef] [PubMed]

17. Cacabelos, R.; Carril, J.C.; Cacabelos, P.; Teijido, O.; Goldgaber, D. Pharmacogenomics of Alzheimer's Disease: Genetic determinants of phenotypic variation and therapeutic outcome. J. Genom. Med. Pharmacogenom. 2016, 1, 151-209.

18. Cacabelos, R.; Fernández-Novoa, L.; Lombardi, V.; Kubota, Y.; Takeda, M. Molecular genetics of Alzheimer's disease and aging. Methods Find. Exp. Clin. Pharmacol. 2005, 27 (Suppl. A), 1-573. [PubMed]

19. Bertram, L.; McQueen, M.B.; Mullin, K.; Blacker, D.; Tanzi, R.E. Systematic meta-analyses of Alzheimer disease genetic association studies: The AlzGene database. Nat. Genet. 2007, 39, 17-23. [CrossRef] [PubMed]

20. Karch, C.M.; Cruchaga, C.; Goate, A. Alzheimer's disease genetics: From the bench to the clinic. Neuron 2014, 83, 11-26. [CrossRef] [PubMed]

21. Schenk, D.; Barbour, R.; Dunn, W.; Gordon, G.; Grajeda, H.; Guido, T.; Hu, K.; Huang, J.; Johnson-Wood, K.; Khan, K.; et al. Immunization with amyloid- $\beta$ attenuates Alzheimer-disease-like pathology in the PDAPP mouse. Nature 1999, 400, 173-177. [CrossRef] [PubMed]

22. Carrera, I.; Etcheverría, I.; Fernández-Novoa, L.; Lombardi, V.; Cacabelos, R.; Vigo, C. Vaccine Development to Treat Alzheimer's Disease Neuropathology in APP/PS1 Transgenic Mice. Int. J. Alzheimers Dis. 2012, 376138. [CrossRef] [PubMed]

23. Carrera, I.; Etcheverría, I.; Fernández-Novoa, L.; Lombardi, V.R.M.; Lakshmana, M.K.; Cacabelos, R.; Vigo, C. A comparative evaluation of a novel vaccine in APP/PS1 mouse models of Alzheimer's Disease. BioMed Res. Int. 2015, 807146. [CrossRef] [PubMed]

24. Cacabelos, R.; Fernández-Novoa, L.; Martínez-Bouza, R.; McKay, A.; Carril, J.C.; Lombardi, V.; Corzo, L.; Carrera, I.; Tellado, I.; Nebril, L.; et al. Future trends in the pharmacogenomics of brain disorders and dementia: Influence of APOE and CYP2D6 variants. Pharmaceuticals 2010, 3, 3040-3100. [CrossRef]

25. Cacabelos, R.; Llovo, R.; Fraile, C.; Fernández-Novoa, L. Pharmacogenetic aspects of therapy with cholinesterase inhibitors: The role of CYP2D6 in Alzheimer's disease pharmacogenetics. Curr. Alzheimer Res. 2007, 4, 479-500. [CrossRef] [PubMed]

26. Cacabelos, R.; Torrellas, C. Epigenetics of aging and Alzheimer's disease: Implications for pharmacogenomics and drug response. Int. J. Mol. Sci. 2015, 16, 30483-30543. [CrossRef] [PubMed] 
27. Clarelli, F.; Mascia, E.; Santangelo, R.; Mazzeo, S.; Giacalone, G.; Galimberti, D.; Fusco, F.; Zuffi, M.; Fenoglio, C.; Franceschi, M.; et al. CHRNA7 Gene and Response to Cholinesterase Inhibitors in an Italian Cohort of Alzheimer's Disease Patients. J. Alzheimers Dis. 2016, 52, 1203-1208. [CrossRef] [PubMed]

28. Yoon, H.; Myung, W.; Lim, S.W.; Kang, H.S.; Kim, S.; Won, H.H.; Carroll, B.J.; Kimet, D.K. Association of the choline acetyltransferase gene with responsiveness to acetylcholinesterase inhibitors in Alzheimer's disease. Pharmacopsychiatry 2015, 48, 111-117. [CrossRef] [PubMed]

29. Braga, I.L.; Silva, P.N.; Furuya, T.K.; Santos, L.C.; Pires, B.C.; Mazzotti, D.R.; Bertolucci, P.H.; Cendoroglo, M.S.; Smith, M.C. Effect of APOE and CHRNA7 genotypes on the cognitive response to cholinesterase inhibitor treatment at different stages of Alzheimer's disease. Am. J. Alzheimers Dis. Other Dement. 2015, 30, 139-144. [CrossRef] [PubMed]

30. Sokolow, S.; Li, X.; Chen, L.; Taylor, K.D.; Rotter, J.I.; Rissman, R.A.; Aisen, P.S.; Apostolova, L.G. Deleterious Effect of Butyrylcholinesterase K-Variant in Donepezil Treatment of Mild Cognitive Impairment. J. Alzheimers Dis. 2017, 56, 229-237. [CrossRef] [PubMed]

31. Martinelli-Boneschi, F.; Giacalone, G.; Magnani, G.; Biella, G.; Coppi, E.; Santangelo, R.; Brambilla, P.; Esposito, F.; Lupoli, S.; Clerici, F.; et al. Pharmacogenomics in Alzheimer's disease: A genome-wide association study of response to cholinesterase inhibitors. Neurobiol. Aging 2013, 34, 1711.e7-1711.e13. [CrossRef] [PubMed]

32. Becquemont, L.; Lecoeur, S.; Simon, T.; Beaune, P.; Funck-Brentano, C.; Jaillon, P. Glutathione S-transferase theta genetic polymorphism might influence tacrine hepatotoxicity in Alzheimer's patients. Pharmacogenetics 1997, 7, 251-253. [CrossRef] [PubMed]

33. Cacabelos, R.; Torrellas, C.; López-Muñoz, F. Epigenomics of Alzheimer's disease. J. Exp. Clin. Med. 2014, 6, 75-82. [CrossRef]

34. Cacabelos, R.; Torrellas, C. Epigenetic drug discovery for Alzheimer's disease. Expert Opin. Drug Discov. 2014, 9, 1059-1086. [CrossRef] [PubMed]

35. Xie, H.G.; Kim, R.B.; Wood, A.J.; Stein, C.M. Molecular basis of ethnic differences in drug disposition and response. Annu. Rev. Pharm. Toxicol. 2001, 41, 815-850. [CrossRef] [PubMed]

36. Cacabelos, R. (Ed.) World Guide for Drug Use and Pharmacogenomics; EuroEspes Publishing Co.: La Coruña, Spain, 2012.

37. Cacabelos, R. Donepezil in Alzheimer's disease: From conventional trials to pharmacogenetics. Neuropsychiatr. Dis. Treat. 2007, 3, 303-333. [PubMed]

38. Cacabelos, R. Pharmacogenomics of central nervous system (CNS) drugs. Drug Dev. Res. 2012, 73, 461-476. [CrossRef]

39. Carril, J.C.; Cacabelos, R. Genomics and Pharmacogenomics of cerebrovascular disorders. J. Genom. Med. Pharmacogenom. 2016, 1, 27-55.

40. Zhao, X.-S.; Peng, J.; Wu, Q.; Zhong, R.; Pan, L.-H.; Tang, Z.-H.; Jiang, Z.-S.; Wang, G.-X.; Liu, L.-S. Imbalanced cholesterol metabolism in Alzheimer's disease. Clin. Chim. Acta 2016, 456, 107-114.

41. Chen, Y.L.; Wang, L.M.; Chen, Y.; Gao, J.Y.; Marshall, C.; Cai, Z.Y.; Hu, G.; Xiao, M. Changes in astrocyte functional markers and $\beta$-amyloid metabolism-related proteins in the early stages of hypercholesterolemia. Neuroscience 2016, 316, 178-191. [CrossRef] [PubMed]

42. Dias, H.K.; Brown, C.L.; Polidori, M.C.; Lip, G.Y.; Griffiths, H.R. LDL-lipids from patients with hypercholesterolaemia and Alzheimer's disease are inflammatory to microvascular endothelial cells: Mitigation by statin intervention. Clin. Sci. 2015, 129, 1195-1206. [CrossRef] [PubMed]

43. Chakrabarti, S.; Khemka, V.K.; Banerjee, A.; Chatterjee, G.; Ganguly, A.; Biswas, A. Metabolic Risk Factors of Sporadic Alzheimer's Disease: Implications in the Pathology, Pathogenesis and Treatment. Aging Dis. 2015, 6, 282-299. [CrossRef] [PubMed]

44. De Oliveira, F.F.; Chen, E.S.; Smith, M.C.; Bertolucci, P.H.F. Pharmacogenetics of angiotensin-converting enzyme inhibitors in patients with Alzheimer's disease dementia. Curr. Alzheimer Res. 2017, 14. [CrossRef] [PubMed]

45. Appleton, J.P.; Scutt, P.; Sprigg, N.; Bath, P.M. Hypercholesterolaemia and vascular dementia. Clin. Sci. 2017, 131, 1561-1578. [CrossRef] [PubMed]

46. Sallustio, F.; Studer, V. Targeting New Pharmacological Approaches for Alzheimer's Disease: Potential for Statins and Phosphodiesterase Inhibitors. CNS Neurol. Disord. Drug Targets 2016, 15, 647-659. [CrossRef] [PubMed] 
47. Sinyavskaya, L.; Gauthier, S.; Renoux, C.; Dell'Aniello, S.; Suissa, S.; Brassard, P. Comparative effect of statins on the risk of incident Alzheimer disease. Neurology 2017. [CrossRef] [PubMed]

48. Daneschvar, H.L.; Aronson, M.D.; Smetana, G.W. Do statins prevent Alzheimer's disease? A narrative review. Eur. J. Intern. Med. 2015, 26, 666-669. [CrossRef] [PubMed]

49. Barone, E.; Di Domenico, F.; Butterfield, D.A. Statins more than cholesterol lowering agents in Alzheimer disease: Their pleiotropic functions as potential therapeutic targets. Biochem. Pharmacol. 2014, 88, $605-616$. [CrossRef] [PubMed]

50. Tamaoka, A. Dyslipidemia and Dementia. Brain Nerve 2016, 68, 737-742. [CrossRef] [PubMed]

51. Hamel, E.; Royea, J.; Ongali, B.; Tong, X.K. Neurovascular and Cognitive failure in Alzheimer's Disease: Benefits of Cardiovascular Therapy. Cell. Mol. Neurobiol. 2016, 36, 219-232. [CrossRef] [PubMed]

52. Samaras, K.; Brodaty, H.; Sachdev, P.S. Does statin use cause memory decline in the elderly? Trends Cardiovasc. Med. 2016, 26, 550-565. [CrossRef] [PubMed]

53. Zissimopoulos, J.M.; Barthold, D.; Brinton, R.D.; Joyce, G. Sex and Race Differences in the Association between Statin Use and the Incidence of Alzheimer Disease. JAMA Neurol. 2016, 74, 225-232. [CrossRef] [PubMed]

54. Zhou, X.; Li, Y.; Shi, X.; Ma, C. An overview on therapeutics attenuating amyloid $\beta$ level in Alzheimer's disease: Targeting neurotransmission, inflammation, oxidative stress and enhanced cholesterol levels. Am. J. Transl. Res. 2016, 8, 246-269. [PubMed]

55. Buxbaum, J.D.; Cullen, E.I.; Friedhoff, L.T. Pharmacological concentrations of the HMGCoA reductase inhibitor lovastatin decrease the formation of the Alzheimer beta-amyloid peptide in vitro and in patients. Front. Biosci. 2002, 7, a50-a59. [PubMed]

56. Yamamoto, N.; Fujii, Y.; Kasahara, R.; Tanida, M.; Ohora, K.; Ono, Y.; Suzuki, K.; Sobue, K. Simvastatin and atorvastatin facilitates amyloid $\beta$-protein degradation in extracellular spaces by increasing neprilysin secretion from astrocytes through activation of MAPK/Erk1/2 pathways. Glia 2016, 64, 952-962. [CrossRef] [PubMed]

57. Cacabelos, R.; Carril, J.C.; Teijido, O. Pharmacogenomics and epigenomics of age-related neurodegenerative disorders: Strategies for drug development. In Anti-Aging Drugs: From Basic Research to Clinical Practice; Vaiserman, A.M., Ed.; RSC Publishing: London, UK, 2017; Volume 57, pp. 75-141.

58. Cacabelos, R.; Vallejo, A.I.; Lombardi, V.; Fernández-Novoa, L.; Pichel, V. E-SAR-94010 (LipoEsar $\left.{ }^{\circledR}\right)$ : A pleiotropic lipoprotein compound with powerful anti-atheromatous and lipid lowering effects. CNS Drug Rev. 2004, 10, 200-201. [CrossRef]

59. Kitzmiller, J.P.; Mikulik, E.B.; Dauki, A.M.; Murkherjee, C.; Luzum, J.A. Pharmacogenomics of statins: Understanding susceptibility to adverse effects. Pharmacogenom. Personal. Med. 2016, 9, 97-106. [CrossRef] [PubMed]

60. Krauss, R.M.; Mangravite, L.M.; Smith, J.D.; Medina, M.W.; Wang, D.; Guo, X.; Rieder, M.J.; Simon, J.A.; Hulley, S.B.; Waters, D.; et al. Variation in the 3-hydroxyl-3-methylglutaryl coenzyme a reductase gene is associated with racial differences in low-density lipoprotein cholesterol response to simvastatin treatment. Circulation 2008, 117, 1537-1544. [CrossRef] [PubMed]

61. Leduc, V.; Bourque, L.; Poirier, J.; Dufour, R. Role of rs3846662 and HMGCR alternative splicing in statin efficacy and baseline lipid levels in familial hypercholesterolemia. Pharmacogenet. Genom. 2016, 26, 1-11. [CrossRef] [PubMed]

62. Peters, B.J.; Rodin, A.S.; Klungel, O.H.; van Duijn, C.M.; Stricker, B.H.; van't Slot, R.; de Boer, A.; Maitland-van der Zee, A.H. Pharmacogenetic interactions between ABCB1 and SLCO1B1 tagging SNPs and the effectiveness of statins in the prevention of myocardial infarction. Pharmacogenomics 2010, 11, 1065-1076. [CrossRef] [PubMed]

63. Keskitalo, J.; Kurkinen, K.; Neuvonen, P.; Niemi, M. ABCB1 haplotypes differentially affect the pharmacokinetics of the acid and lactone forms of simvastatin and atorvastatin. Clin. Pharmacol. Ther. 2008, 84, 457-461. [CrossRef] [PubMed]

64. Wang, D.; Guo, Y.; Wrughton, S.A.; Cooke, G.E.; Sadee, W. Intronic polymorphism in CYP3A4 affects hepatic expression and response to statin drugs. Pharmacogenom. J. 2011, 11, 274-286. [CrossRef] [PubMed] 
65. Westlind-Johnsson, A.; Malmebo, S.; Johansson, A.; Otter, C.; Andersson, T.B.; Johansson, I.; Edwards, R.J.; Boobis, A.R.; Ingelman-Sundberg, M. Comparative analysis of CYP3A4 expression in human liver suggests only a minor role for CYP3A5 in drug metabolism. Drug Metab. Dispos. 2003, 31, 755-761. [CrossRef] [PubMed]

66. Kim, K.A.; Park, P.W.; Lee, O.J.; Kang, D.K.; Park, J.Y. Effect of polymorphic CYP3A5 genotype on the single-dose simvastatin pharmacokinetics in healthy subjects. J. Clin. Pharmacol. 2007, 47, 87-93. [CrossRef] [PubMed]

67. Norton, S.; Matthews, F.E.; Barnes, D.E.; Yaffe, K.; Brayne, C. Potential for primary prevention of Alzheimer's disease: An analysis of population-based data. Lancet Neurol. 2014, 13, 788-794. [CrossRef]

68. Moonga, I.; Niccolini, F.; Wilson, H.; Pagano, G.; Politis, M. Alzheimer's Disease Neuroimaging Initiative. Hypertension is associated with worse cognitive function and hippocampal hypometabolism in Alzheimer's disease. Eur. J. Neurol. 2017, 24, 1173-1182. [CrossRef] [PubMed]

69. Wain, L.V.; Vaez, A.; Jansen, R.; Joehanes, R.; van der Most, P.J.; Erzurumluoglu, A.M.; O’Reilly, P.F.; Cabrera, C.P.; Warren, H.R.; Rose, L.M.; et al. Novel Blood Pressure Locus and Gene Discovery Using Genome-Wide Association Study and Expression Data Sets From Blood and the Kidney. Hypertension 2017, 70, e4-e19. [CrossRef] [PubMed]

70. Edwards, J.D.; Ramirez, J.; Callahan, B.L.; Tobe, S.W.; Oh, P.; Berezuk, C.; Lanctôt, K.; Swardfager, W.; Nestor, S.; Kiss, A.; et al. Alzheimer's Disease Neuroimaging Initiative. Antihypertensive Treatment is associated with MRI-Derived Markers of Neurodegeneration and Impaired Cognition: A Propensity-Weighted Cohort Study. J. Alzheimers Dis. 2017, 59, 1113-1122. [CrossRef] [PubMed]

71. Bhat, S.A.; Goel, R.; Shukla, S.; Shukla, R.; Hanif, K. Angiotensin Receptor Blockade by Inhibiting Glial Activation Promotes Hippocampal Neurogenesis via Activation of Wnt/ $\beta$-Catenin Signaling in Hypertension. Mol. Neurobiol. 2017. [CrossRef] [PubMed]

72. Sundbøll, J.; Hováth-Puhó, E.; Adelborg, K.; Schmidt, M.; Pedersen, L.; Bøtker, H.E.; Henderson, V.W.; Sørensen, H.T. Higher Risk of Vascular Dementia in Myocardial Infarction Survivors. Circulation 2017. [CrossRef] [PubMed]

73. Schievink, S.H.J.; van Boxtel, M.P.J.; Deckers, K.; van Oostenbrugge, R.J.; Verhey, F.R.J.; Köhler, S. Cognitive changes in prevalent and incident cardiovascular disease: A 12-year follow-up in the Maastricht Aging Study (MAAS). Eur. Heart J. 2017, ehx365. [CrossRef] [PubMed]

74. Gabin, J.M.; Tambs, K.; Saltvedt, I.; Sund, E.; Holmen, J. Association between blood pressure and Alzheimer disease measured up to 27 years prior to diagnosis: The HUNT Study. Alzheimers Res. Ther. 2017, 9, 37. [CrossRef] [PubMed]

75. Kern, K.C.; Wright, C.B.; Bergfield, K.L.; Fitzhugh, M.C.; Chen, K.; Moeller, J.R.; Nabizadeh, N.; Elkind, M.S.V.; Sacco, R.L.; Stern, Y.; et al. Blood Pressure Control in Aging Predicts Cerebral Atrophy Related to Small-Vessel White Matter Lesions. Front. Aging Neurosci. 2017, 9, 132. [CrossRef] [PubMed]

76. Cacabelos, R. Epigenomic networking in drug development: From pathogenic mechanisms to pharmacogenomics. Drug Dev. Res. 2014, 75, 348-365. [CrossRef] [PubMed]

(c) 2018 by the authors. Licensee MDPI, Basel, Switzerland. This article is an open access article distributed under the terms and conditions of the Creative Commons Attribution (CC BY) license (http:/ / creativecommons.org/licenses/by/4.0/). 